\title{
Pessimistic Multigranulation Rough Set of Intuitionistic Fuzzy Sets Based on Soft Relations
}

\author{
Muhammad Zishan Anwar ${ }^{1}$, Ahmad N. Al-Kenani ${ }^{2}\left(\mathbb{D}\right.$, Shahida Bashir ${ }^{1, *(D)}$ and Muhammad Shabir ${ }^{3}$ \\ 1 Department of Mathematics, University of Gujrat, Gujrat 50700, Pakistan; 18026109-002@uog.edu.pk \\ 2 Department of Mathematics, Faculty of Science, King Abdulaziz University, P.O. Box 80219, \\ Jeddah 21589, Saudi Arabia; analkenani@kau.edu.sa \\ 3 Department of Mathematics, Quaid-I-Azam University, Islamabad 45320, Pakistan; \\ mshabirbhatti@qau.edu.pk \\ * Correspondence: shahida.bashir@uog.edu.pk
}

check for

updates

Citation: Anwar, M.Z.; Al-Kenani,

A.N.; Bashir, S.; Shabir, M. Pessimistic Multigranulation Rough Set of Intuitionistic Fuzzy Sets Based on Soft Relations. Mathematics 2022, 10, 685. https://doi.org/10.3390/ math10050685

Academic Editors: Zhen-Song Chen, Witold Pedrycz, Lesheng Jin, Rosa M. Rodriguez and Luis Martínez López

Received: 4 January 2022

Accepted: 16 February 2022

Published: 22 February 2022

Publisher's Note: MDPI stays neutral with regard to jurisdictional claims in published maps and institutional affiliations.

Copyright: (c) 2022 by the authors. Licensee MDPI, Basel, Switzerland. This article is an open access article distributed under the terms and conditions of the Creative Commons Attribution (CC BY) license (https:/ / creativecommons.org/licenses/by/ $4.0 /)$.

\begin{abstract}
Qian presented multigranulation rough set (MGRS) models based on Pawlak's rough set (RS) model. There are two types of MGRS models, named optimistic MGRS and pessimistic MGRS. Recently, Shabir et al. presented an optimistic multigranulation intuitionistic fuzzy rough set (OMGIFRS) based on soft binary relations. This paper explores the pessimistic multigranulation intuitionistic fuzzy rough set (PMGIFRS) based on soft relations combined with a soft set (SS) over two universes. The resulting two sets are lower approximations and upper approximations with respect to the aftersets and foresets. Some basic properties of this established model are studied. Similarly, the MGRS of an IFS based on multiple soft relations is presented and some algebraic properties are discussed. Finally, an example is presented that illustrates the importance of the proposed decision-making algorithm.
\end{abstract}

Keywords: intuitionistic fuzzy set; soft relation; multigranulation roughness; decision making

\section{Introduction}

Naturally, we face several critical situations that have vagueness and uncertainty. In today's fast-paced life, we need methods and techniques through which we manage this uncertainty in a better way. Mathematicians have shown interest in overcoming these situations and have presented several theories, such as fuzzy set (FS) theory, IFS theory, SS theory, RS theory, etc.

Zadeh [1] built a framework of FSs to manage uncertainty, vagueness and impreciseness. For several research purposes, FSs are useful, ingenious and innovative sets. The FSs are very helpful sets to solve real world problems due to qualitative expressions. Each element of FS is described with its degree of membership. However, often, we face many situations involving vagueness and impreciseness that can not be controlled by a FS due to its degree of membership only. For example, a doctor wants to diagnose a disease in a patient but the disease can not be diagnosed by the membership degree only. To manage such situations, Attanasov presented an IFS [2], which is extension of a FS, and each element of an IFS is described with a membership degree, non-membership degree and hesitant degree. IFSs are very useful sets, and applications of IFSs in several fields show their importance. The IFSs have many applications in market prediction, the electoral system, career determination, medical diagnosis and machine learning [3]. The IFSs have many operations and properties that help to manage impreciseness and uncertainty better than FSs.

Many new models, such as FS (1965), RS (1982) and IFS (1986), have been introduced to control uncertainty, which is an inherent characteristic of modern databases. All of these models have their own algebraic properties and operations but these models lack a sufficient number of parameters to control uncertainty. To solve this problem, Molodtsov (1999) [4] 
introduced the SS, which is an untraditional approach to deal with uncertainty. SSs have an adequate number of parameters and many operations. SSs are also helpful in decision making, data clustering, parameter reduction and data to deal with incompleteness. Many extensions of SSs have been introduced, such as vague SS [5], fuzzy SS (FSS) [6], IF soft set (IFSS) [7], soft RS (SRS) [8], trapezoidal FSS [9], fuzzy RS (FRS), etc. Sang et al. [10] presented an intuitionistic fuzzy rough set (IFRS) model to deal with the unnaturalness of FRS.

Pawlak (1982) [11] invented the RS, which is an untraditional approach to deal with inconsistent, imprecise and incomplete knowledge. The RS theory can be divided into two major parts as a simple information model. The first part concerns the rules and concepts formed by the classification of the relational database, and the second part concerns the innovative knowledge discovered by the equivalence relation and approximation of the target. The RS theory is a better theory than the FS theory because the FS has a degree that is uncertain and the RS establishes two precise boundary lines for a description of imprecise concepts [12]. The RS theory is a useful tool in the intelligent information processing field. Many extensions of RS models have been developed recently. Combining RS theory with existing theories, many generalizations have been introduced, such as probabilistic RSs [13], a decision theoretic RS model, a game theoretic RS model [14], etc. The RS model has rich applications in several fields of modern research. In theoretical research, the RS model describes algebraic structures with abstract algebra [15], rough approximation topology [16] and a combination of a soft computing method with RS theory [17]. In application research, the RS model is helpful in medical diagnosis [18], image processing [19], intelligent analysis [20], E-mail filtering [21], etc.

The multigranulation rough set (MGRS) is a very helpful technique for the description of problem solving. In the MGRS, multiple relations are used, which is advantageous as a collective decision based on multiple experts' opinions is wiser than a single expert's opinion. Granular computing is a very useful method to make better decisions in complicated real world problems nowadays. In 2010, Qian [22] introduced an extension of the RS model in terms of the MGRS model for the first time. Comparing with the classical RS model, the MGRS model is proposed, and the resulting sets are smaller in lower approximation sets and bigger in upper approximation sets. Qian [23] introduced two types of MGRS models: optimistic MGRS (OMGRS) and pessimistic MGRS (PMGRS). Covering the MGRS [24] is useful in dealing with data sets that involve overlapping and a large amount of knowledge. After that, MGRSs with generalized relations [25], order relations [26] and fuzzy compatible relations [27] have been discussed by relaxing the condition of equivalence relations. Different researchers modified MGRS models to manage different data set needs. According to the nature of problems in several fields, the MGRS showed its significance and importance [28-32]. The risk attitudes by OMGRS and PMGRS models based on multiple relations have been discussed by Qian et al. [33,34]. Huang et al. [35] built a suitable framework of a combination of MGRS with an IFS, which was named the IFMGRS model. Pang et al. [36] described the combination with three-way decision making and proposed a multi-criteria decision-making model. Sun and Ma [37] presented a MGRS model in connection with two universes. Tan et al. [38] combined a MGRS model and a granularity selection algorithm to make a selective data set approximation. Xu et al. [39] built a framework of a combination of the RS model, MGRS model and FRS model in connection with granular computing, and this combination is said to be a MGFRS. Shabir et al. [40] presented a MGRS model based on multi soft relations. Recently, the extension of the MGRS in terms of the FS has been presented by Shabir et al., and is called the OMFGRS. After that, Shabir et al. [41-43] presented an optimistic multigranulation intuitionistic fuzzy rough set. They discussed the basic properties of the OMIFGRS in [41]. Now, we discuss the PMGRS in terms of the IFS based on soft relations.

In our realistic world, multiple universes of objects are needed in several practical problems, such as medication and disease symptoms used in disease diagnostics. Since Pawlak discussed the RS model using a single universe, to solve the above problem about 
multiple universes of objects, Yan et al. and Liu discussed the RS model using dual universes. They presented a comparative study of the RS model based on single universe and dual universes. The RS model based on dual universes is very useful in dealing with multi-granulation information. Qian et al. extended the RS model based on dual universes and presented the MGRS to approximate a set based on a finite number of relations.

In many fields, the contribution of MGRS is significant, such as in conflict analysis problems, in medical diagnostics, in decision-making problems, in steam turbine defect diagnostic models, in granulation selection and in decision making with MGRS based on dual universes. Shabir et al. discussed the RS model based on dual universes and the MGRS based on multiple universes. They investigated a pessimistic MGRS of a FS in terms of soft relations. Since the FS discusses the degree of membership only, but we have to face many problems in the real world that have uncertainty and vagueness, the FS is not applicable in many complicated situations. As a result of this problem, the IFS is more useful in dealing with critical situations due to its membership degree and non-membership degree. This is the main idea for the motivation of our research work.

The rest of the paper is organized as follows. Section 2 presents the FS, SS, RS, IFS, IFSS, MGRS and soft binary relations. In Section 3, a pessimistic MGRS of an IFS based on two soft binary relations over dual universes and its properties are given. Section 4 presents the PMGIFRS over dual universes and their algebraic properties with examples. In Section 5, a decision-making algorithm of this proposed model with a practical example is presented. Section 6 presents a comparative study of our proposed model and other existing theories. Finally, the conclusion of our research work is described in Section 7.

\section{Preliminaries and Basic Concepts}

This section presents some basic notions about the RS, IFS, SS, MGRS, soft binary relation and IFSS. Throughout this paper, $W_{1}$ and $W_{2}$ represent two non-empty finite sets unless stated otherwise.

Definition 1 ([2]). Let $W$ be a non-empty universe. An IFS $T$ in the universe $W$ is an object having the form $T=\left\{\left\langle x, \mu_{T}(x), \gamma_{T}(x)\right\rangle: x \in W\right\}$, where $\mu_{T}: W \rightarrow[0,1]$ and $\gamma_{T}: W \rightarrow[0,1]$ satisfying $0 \leq \mu_{T}(x)+\gamma_{T}(x) \leq 1$ for all $x \in W$. The values $\mu_{T}(x)$ and $\gamma_{T}(x)$ are called the degree of membership and degree of non-membership of $x \in W$ to $T$, respectively. The number $\pi_{T}(x)=1-\mu_{T}(x)-\gamma_{T}(x)$ is called the degree of hesitancy of $x \in W$ to $T$. The collection of all IFSs in $W$ is denoted by IF $(W)$. In the remaining paper, we shall write an IFS by $T=\left\langle\mu_{T}, \gamma_{T}\right\rangle$ instead of $T=\left\{\left\langle x, \mu_{T}(x), \gamma_{T}(x)\right\rangle: x \in W\right\}$. Let $T=\left\langle\mu_{T}, \gamma_{T}\right\rangle$ and $T_{1}=\left\langle\mu_{T_{1}}, \gamma_{T_{1}}\right\rangle$ be two IFSs in $W$. Then, $T \subseteq T_{1}$ if and only if $\mu_{T}(x) \leq \mu_{T_{1}}(x)$ and $\gamma_{T_{1}}(x) \leq \gamma_{T}(x)$ for all $x \in W$. Two IFSs $T$ and $T_{1}$ are said to be equal if and only if $T \subseteq T_{1}$ and $T_{1} \subseteq T$.

Definition 2 ([2]). The union and intersection of two IFSs $T$ and $T_{1}$ in $W$ are denoted and defined by $T \cup T_{1}=\left\langle\mu_{T} \cup \mu_{T_{1}}, \gamma_{T} \cap \gamma_{T_{1}}\right\rangle$ and $T \cap T_{1}=\left\langle\mu_{T} \cap \mu_{T_{1}}, \gamma_{T} \cup \gamma_{T_{1}}\right\rangle$, where $\left(\mu_{T} \cup \mu_{T_{1}}\right)(x)=$ $\sup \left\{\mu_{T}(x), \mu_{T_{1}}(x)\right\},\left(\gamma_{T} \cap \gamma_{T_{1}}\right)(x)=\inf \left\{\gamma_{T}(x), \gamma_{T_{1}}(x)\right\}$, for all $x \in W$.

Next, we define two special types of IFSs as:

The IF universe set $W=1_{W}=<1,0>$ and IF empty set $\Phi=0_{W}=<0,1>$, where $1(x)=1$ and $0(x)=0$ for all $x \in W$. The complement of an IFS $T=\langle\mu, \gamma>$ is denoted and defined as $T^{\mathcal{C}}=<\gamma, \mu>$.

For a fixed $x \in W$, the pair $\left(\mu_{T}(x), \gamma_{T}(x)\right)$ is called the intuitionistic fuzzy value (IFV) or intuitionistic fuzzy number (IFN). In order to define the order between two IFNs, Chen and Tan [44] introduced the score function as $S(x)=\mu_{T}(x)-\gamma_{T}(x)$ and Hong and Choi [45] defined the accuracy function as $H(x)=\mu_{T}(x)+\gamma_{T}(x)$, where $x \in W$. Xu [39] used both the score and accuracy functions to define the order relation between any pair $(x, y)$ of IFVS as given below:

(a) If $S(x)>S(y)$, then $x>y$

(b) If $S(x)=S(y)$, then 
(1) If $H(x)=H(y)$, then $x=y$

(2) If $H(x)<H(y)$, then $x<y$.

Definition 3 ([11]). Let $\lambda$ be an equivalence relation on a universe $W$. For any $P \subseteq W$, the Pawlak lower and upper approximations of $P$ with respect to $\lambda$ are defined by

$$
\begin{aligned}
& \underline{\lambda}(P)=\left\{w \in W:[w]_{\lambda} \subseteq P\right\}, \\
& \bar{\lambda}(P)=\left\{w \in W:[w]_{\lambda} \cap P \neq \varnothing\right\} .
\end{aligned}
$$

where $[w]_{\lambda}$ is the equivalence class of $w$ with respect to $\lambda$. The set $B P_{\lambda}=\bar{\lambda}(P)-\underline{\lambda}(P)$ is the boundary region of $P \subseteq W$. If $B P_{\lambda}(P)=\varnothing$, then $P$ is defineable (exact); otherwise, $P$ is rough with respect to $\lambda$.

Qian et al. [23] extended the Pawlak rough set model to the MGRS model, where the set approximations are defined by using multi-equivalence relations on a universe.

Definition 4 ([23]). Let $\lambda_{1}, \lambda_{2}, \lambda_{3}, \ldots . ., \lambda_{n}$ be $m$ equivalence relations on a universe $W$. For any $P \subseteq W$, the Pawlak lower and upper approximations of $P$ are defined by

$$
\begin{aligned}
& \underline{P}_{\sum_{i=1}^{m} \lambda_{i}}=\left\{w \in W:[w]_{\lambda_{i}} \subseteq P \text { for some } i, 1 \leq i \leq m\right\} \\
& \bar{P}^{\sum_{i=1}^{m} \lambda_{i}}=\left(\underline{P^{c}} \sum_{i=1}^{m} \lambda_{i}\right)^{c} .
\end{aligned}
$$

where $[w]_{\lambda_{i}}$ is the equivalence class of $w$ with respect to $\lambda_{i}$

Definition 5 ([46]). A pair $(\lambda, A)$ is called a SS over $W$ if $\lambda$ is a mapping given by $\lambda: A \rightarrow P(W)$, where $A$ is a subset of $E$ (the set of parameters) and $P(W)$ is the power set of $W$. Thus, $\lambda(e)$ is a subset of $W$ for all $e \in A$. Hence, a SS over $W$ is a parametrized collection of subsets of $W$.

Definition $6([47])$. Let $(\lambda, A)$ be a SS over $W_{1} \times W_{1}$. Then, $(\lambda, A)$ is called a soft binary relation on $W_{1}$. In fact, $(\lambda, A)$ is a parameterized collection of binary relations on $W_{1}$; that is, we have a binary relation $\lambda(e)$ on $W_{1}$ for each parameter $e \in A$.

Li et al. [48] presented the generalization of soft binary relation from $W_{1}$ to $W_{2}$ as follows.

Definition 7 ([48]). A soft binary relation $(\lambda, A)$ from $W_{1}$ to $W_{2}$ is a SS over $W_{1} \times W_{2}$; that is, $\lambda: A \rightarrow P\left(W_{1} \times W_{2}\right)$, where $A$ is a subset of the set of parameters $E$.

Of course, $(\lambda, A)$ is a parameterized collection of binary relations from $W_{1}$ to $W_{2}$. That is, for each $e \in A$, we have a binary relation $\lambda(e)$ from $W_{1}$ to $W_{2}$.

Definition 8 ([7]). A pair $(\lambda, A)$ is called an IFSS over $W$ if $\lambda$ is a mapping given by $\lambda: A \rightarrow$ $\operatorname{IF}(W)$ and $A$ is a subset of $E$ (the set of parameters). Thus, $\lambda(e)$ is an IFS in $W$ for all $e \in A$. Hence, an IFSS over $W$ is a parametrized collection of IF sets in $W$.

Definition 9 ([7]). For two IFSSs $(\lambda, A)$ and $(\rho, B)$ over a common universe $W$, we say that $(\lambda, A)$ is an IF soft subset of $(\rho, B)$ if $(1) A \subseteq B$, and $(2) \lambda(e)$ is an IF subset of $\rho(e)$ for all $e \in A$. Two IFSSs $(\lambda, A)$ and $(\rho, B)$ over a common universe $W$ are said to be IF soft equal if $(\lambda, A)$ is an IF soft subset of $(\rho, B)$ and $(\rho, B)$ is an IF soft subset of $(\lambda, A)$. The union of two IFSSs $(\lambda, A)$ and $(\rho, A)$ over the common universe $W$ is the IFSS $(H, A)$, where $H(e)=\lambda(e) \cup \rho(e)$ for all $e \in A$. The intersection of two IFSSs $(\lambda, A)$ and $(\rho, A)$ over the common universe $W$ is the IFSS $(K, A)$, where $K(e)=\lambda(e) \cap \rho(e)$ for all $e \in A$.

Definition 10 ([49]). Let $(\lambda, A)$ be a soft binary relation from $W_{1}$ to $W_{2}$ and $T=\left\langle\mu_{T}, \gamma_{T}\right\rangle$ be an IFS in $W_{2}$. Then, the lower approximation $\underline{\lambda}^{T}=\left(\underline{\lambda}^{\mu_{T}}, \underline{\lambda}^{\gamma_{T}}\right)$ and upper approximation $\bar{\lambda}^{T}=\left(\bar{\lambda}^{\mu_{T}}, \bar{\lambda}^{\gamma_{T}}\right)$ of $T=\left\langle\mu_{T}, \gamma_{T}\right\rangle$ with respect to aftersets are defined as follows: 


$$
\begin{aligned}
& \underline{\lambda}^{\mu_{T}}(e)\left(w_{1}\right)=\left\{\begin{array}{cc}
\wedge_{a \in w_{1} \lambda(e)} \mu_{T}(a) & \text { if } w_{1} \lambda(e) \neq \varnothing ; \\
1 & \text { if } w_{1} \lambda(e)=\varnothing ;
\end{array}\right. \\
& \underline{\lambda}^{\gamma_{T}}(e)\left(w_{1}\right)=\left\{\begin{array}{cc}
\vee_{a \in w_{1} \lambda(e)} \gamma_{T}(a) & \text { if } w_{1} \lambda(e) \neq \varnothing ; \\
0 & \text { if } w_{1} \lambda(e)=\varnothing ;
\end{array}\right.
\end{aligned}
$$

and

$$
\begin{aligned}
& \bar{\lambda}^{\mu_{T}}(e)\left(w_{1}\right)=\left\{\begin{array}{cc}
\vee_{a \in w_{1} \lambda(e)} \mu_{T}(a) & \text { if } w_{1} \lambda(e) \neq \varnothing ; \\
0 & \text { if } w_{1} \lambda(e)=\varnothing ;
\end{array}\right. \\
& \bar{\lambda}^{\gamma_{T}}(e)\left(w_{1}\right)=\left\{\begin{array}{cc}
\wedge_{a \in w_{1} \lambda(e)} \gamma_{T}(a) & \text { if } w_{1} \lambda(e) \neq \varnothing ; \\
1 & \text { if } w_{1} \lambda(e)=\varnothing .
\end{array}\right.
\end{aligned}
$$

where $w_{1} \lambda(e)=\left\{a \in W_{2}:\left(w_{1}, a\right) \in \lambda(e)\right\}$ and is called the afterset of $w_{1}$ for $w_{1} \in W_{1}$ and $e \in A$.

- $\quad \underline{\lambda}^{\mu_{T}}(e)\left(w_{1}\right)$ indicates the degree to which $w_{1}$ definitely has the property $e$;

- $\quad \underline{\lambda}^{\gamma_{T}}(e)\left(w_{1}\right)$ indicates the degree to which $w_{1}$ probably does not have the property $e$;

- $\quad \bar{\lambda}^{\mu_{T}}(e)\left(w_{1}\right)$ indicates the degree to which $w_{1}$ probably has the property $e$;

- $\quad \bar{\lambda}^{\gamma_{T}}(e)\left(w_{1}\right)$ indicates the degree to which $w_{1}$ definitely does not have the property $e$.

Definition 11 ([49]). Let $(\lambda, A)$ be a soft binary relation from $W_{1}$ to $W_{2}$ and $T=\left\langle\mu_{T}, \gamma_{T}\right\rangle$ be an IFS in $W_{1}$. Then, the lower approximation ${ }^{T} \underline{\lambda}=\left(\mu_{T} \underline{\lambda}, \gamma_{T} \underline{\lambda}\right)$ and upper approximation $T \bar{\lambda}=\left(\mu_{T} \bar{\lambda}, \gamma_{T} \bar{\lambda}\right)$ of $T=\left\langle\mu_{T}, \gamma_{T}\right\rangle$ with respect to foresets are defined as follows:

$$
\begin{gathered}
\mu_{T} \underline{\lambda}(e)\left(w_{2}\right)=\left\{\begin{array}{cc}
\wedge_{a \in \lambda(e) w_{2}} \mu_{T}(a) & \text { if } \lambda(e) w_{2} \neq \varnothing ; \\
1 & \text { if } \lambda(e) w_{2}=\varnothing ;
\end{array}\right. \\
\gamma_{T} \underline{\lambda}(e)\left(w_{2}\right)=\left\{\begin{array}{cc}
\vee_{a \in \lambda(e) w_{2}} \gamma_{T}(a) & \text { if } \lambda(e) w_{2} \neq \varnothing ; \\
0 & \text { if } \lambda(e) w_{2}=\varnothing ;
\end{array}\right.
\end{gathered}
$$

and

$$
\begin{aligned}
& \mu_{T} \bar{\lambda}(e)\left(w_{2}\right)=\left\{\begin{array}{cc}
\vee_{a \in \lambda(e) w_{2}} \mu_{T}(a) & \text { if } \lambda(e) w_{2} \neq \varnothing ; \\
0 & \text { if } \lambda(e) w_{2}=\varnothing ;
\end{array}\right. \\
& \gamma_{T} \bar{\lambda}(e)\left(w_{2}\right)=\left\{\begin{array}{cc}
\wedge_{a \in \lambda(e) w_{2}} \gamma_{T}(a) & \text { if } \lambda(e) w_{2} \neq \varnothing ; \\
1 & \text { if } \lambda(e) w_{2}=\varnothing .
\end{array}\right.
\end{aligned}
$$

where $\lambda(e) w_{2}=\left\{a \in W_{1}:\left(a, w_{2}\right) \in \lambda(e)\right\}$ and is called the foreset of $w_{2}$ for $w_{2} \in W_{2}$ and $e \in A$.

Ofcourse, $\underline{\lambda}^{T}: A \rightarrow \operatorname{IF}\left(W_{1}\right), \bar{\lambda}^{T}: A \rightarrow \operatorname{IF}\left(W_{1}\right)$ and ${ }^{T} \underline{\lambda}: A \rightarrow \operatorname{IF}\left(W_{2}\right),{ }^{T} \bar{\lambda}: A \rightarrow$ $\operatorname{IF}\left(W_{2}\right)$.

Theorem 1 ([49]). Let $(\lambda, A)$ be a soft binary relation from $W_{1}$ to $W_{2}$; that is, $\lambda: A \rightarrow P\left(W_{1} \times\right.$ $\left.W_{2}\right)$. For any IFS, $T=\left\langle\mu_{T}, \gamma_{T}\right\rangle, T_{1}=\left\langle\mu_{T_{1}}, \gamma_{T_{1}}\right\rangle$ and $T_{2}=\left\langle\mu_{T_{2}}, \gamma_{T_{2}}\right\rangle$ of $W_{2}$, the following are true:

(1) If $T_{1} \subseteq T_{2}$ then $\underline{\lambda}^{T_{1}} \subseteq \underline{\lambda}^{T_{2}}$;

(2) If $T_{1} \subseteq T_{2}$ then $\bar{\lambda}^{T_{1}} \subseteq \bar{\lambda}^{T_{2}}$;

(3) $\lambda^{T_{1}} \cap \lambda^{T_{2}}=\lambda^{T_{1} \cap T_{2}}$;

(4) $\bar{\lambda}^{T_{1}} \cap \bar{\lambda}^{T_{2}} \supseteq \bar{\lambda}^{T_{1} \cap T_{2}}$;

(5) $\underline{\lambda}^{T_{1}} \cup \underline{\lambda}^{T_{2}} \subseteq \underline{\lambda}^{T_{1} \cup T_{2}}$;

(6) $\bar{\lambda}^{T_{1}} \cup \bar{\lambda}^{T_{2}}=\bar{\lambda}^{T_{1} \cup T_{2}}$;

(7) $\underline{\lambda}^{1 W_{2}}=1 W_{1}$ if $w_{1} \lambda(e) \neq \varnothing$;

(8) $\bar{\lambda}^{-1} W_{2}=1 W_{1}$ if $w_{1} \lambda(e) \neq \varnothing$;

(9) $\underline{\lambda}^{T}=\left(\bar{\lambda}^{T^{c}}\right)^{c}$ if $w_{1} \lambda(e) \neq \varnothing$; 
(10) $\bar{\lambda}^{T}=\left(\underline{\lambda}^{T^{c}}\right)^{c}$ if $w_{1} \lambda(e) \neq \varnothing$;

(11) $\underline{\lambda}^{0_{W_{2}}}=0_{W_{1}}=\bar{\lambda}^{0_{W_{2}}}$ if $w_{1} \lambda(e) \neq \varnothing$.

Proof. This is proved in [49].

Theorem 2 ([49]). Let $(\lambda, A)$ be a soft binary relation from $W_{1}$ to $W_{2}$; that is, $\lambda: A \rightarrow P\left(W_{1} \times\right.$ $\left.W_{2}\right)$. For any IFS, $T=\left\langle\mu_{T}, \gamma_{T}\right\rangle, T_{1}=\left\langle\mu_{T_{1}}, \gamma_{T_{1}}\right\rangle$ and $T_{2}=\left\langle\mu_{T_{2}}, \gamma_{T_{2}}\right\rangle$ of $W_{1}$, the following are true:

(1) If $T_{1} \subseteq T_{2}$ then ${ }^{T_{1}} \underline{\underline{\lambda}} \subseteq^{T_{2}} \underline{\lambda}$;

(2) If $T_{1} \subseteq T_{2}$ then $T_{1} \bar{\lambda} \subseteq T_{2} \bar{\lambda}$;

(3) $T_{1} \underline{\lambda} \cap \cap^{T_{2}} \underline{\lambda}==_{1}^{T_{1} \cap T_{2}} \underline{\lambda}$;

(4) $\lambda_{1} \overline{\bar{\lambda}} \cap 2 \bar{\lambda} \supseteq \bigcap_{1} \cap T_{2} \bar{\lambda}$;

(5) $T_{1} \lambda \cup T^{T_{2}} \lambda \subseteq T_{1} \cup T_{2} \lambda$;

(6) $T_{1} \bar{\lambda} \cup T_{2} \bar{\lambda}=T_{1} \cup T_{2} \bar{\lambda}$;

(7) $1_{W_{1}} \underline{\lambda}=1_{W_{2}}$ if $\lambda(e) w_{2} \neq \varnothing$;

(8) $1_{W_{1}} \bar{\lambda}=1_{W_{2}}$ if $\lambda(e) w_{2} \neq \varnothing$;

(9) ${ }^{T} \underline{\lambda}=\left(T^{c} \bar{\lambda}\right)^{c}$ if $\lambda(e) w_{2} \neq \varnothing$;

(10) $T \bar{\lambda}=\left(T^{c} \underline{\lambda}\right)^{c}$ if $\lambda(e) w_{2} \neq \varnothing$;

(11) ${ }^{0}{ }_{W_{1}} \underline{\lambda}=0_{W_{2}}={ }^{0}{ }_{W_{1}} \bar{\lambda}$.

Proof. This is proved in [49].

\section{Roughness of an Intuitionistic Fuzzy Set by Two Soft Relations}

In this section, the pessimistic multigranulation roughness of an IFS by two soft binary relations from $W_{1}$ to $W_{2}$ has been discussed. We approximate an IFS of universe $W_{2}$ in universe $W_{1}$ and an IFS of $W_{1}$ in $W_{2}$ by using aftersets and foresets of soft binary relations, respectively. In this way, we obtain two IFSSs corresponding to IFSs in $W_{2}\left(W_{1}\right)$. We also discuss some properties of these approximations.

Definition 12. Let $W_{1}$ and $W_{2}$ be two non-empty sets, $\left(\lambda_{1}, A\right)$ and $\left(\lambda_{2}, A\right)$ be two soft binary relations from $W_{1}$ to $W_{2}$ and $T=\left\langle\mu_{T}, \gamma_{T}\right\rangle$ be an IFS in $W_{2}$. Then, the pessimistic multigranulation lower approximation $\underline{\lambda_{1}+\lambda_{2}^{T}}=\left(\underline{\lambda_{1}+\lambda_{2}}{ }_{p}^{\mu_{T}}, \underline{\lambda}_{1}+\lambda_{2}^{\gamma_{T}}\right)$ and the pessimistic multigranulation upper approximation $p{\overline{\lambda_{1}+\lambda_{2}}}^{T}=\left(p{\overline{\lambda_{1}+\lambda_{2}}}^{\mu_{T}}, p{\overline{\lambda_{1}+\lambda_{2}}}^{\gamma}\right)$ of $T=\left\langle\mu_{T}, \gamma_{T}\right\rangle$ are IF soft sets over $W_{1}$ and are defined as:

$$
\begin{aligned}
& {\underline{\lambda_{1}+\lambda_{2}^{\mu_{T}}}}_{p}^{\mu_{1}}(e)\left(w_{1}\right)=\left\{\begin{array}{cc}
\wedge\left\{\mu_{T}\left(w_{2}\right): w_{2} \in\left(w_{1} \lambda_{1}(e) \cap w_{1} \lambda_{2}(e)\right)\right\}, & \text { if } w_{1} \lambda_{1}(e) \cap w_{1} \lambda_{2}(e) \neq \varnothing ; \\
1 & \text { otherwise; }
\end{array}\right. \\
& \underline{\lambda_{1}+\lambda_{2}^{\gamma}} \gamma_{T}(e)\left(w_{1}\right)=\left\{\begin{array}{cc}
\vee\left\{\gamma_{T}\left(w_{2}\right): w_{2} \in\left(w_{1} \lambda_{1}(e) \cup w_{1} \lambda_{2}(e)\right)\right\}, & \text { if } w_{1} \lambda_{1}(e) \cup w_{1} \lambda_{2}(e) \neq \varnothing ; \\
0 & \text { otherwise; }
\end{array}\right.
\end{aligned}
$$

and

$p{\overline{\lambda_{1}+\lambda_{2}}}^{\mu_{T}}(e)\left(w_{1}\right)=\left\{\begin{array}{cc}\vee\left\{\mu_{T}\left(w_{2}\right): w_{2} \in\left(w_{1} \lambda_{1}(e) \cup w_{1} \lambda_{2}(e)\right)\right\}, & \text { if } w_{1} \lambda_{1}(e) \cup w_{1} \lambda_{2}(e) \neq \varnothing ; \\ 0 & \text { otherwise; }\end{array}\right.$

$p{\overline{\lambda_{1}+\lambda_{2}}}^{\gamma}(e)\left(w_{1}\right)=\left\{\begin{array}{cc}\wedge\left\{\gamma_{T}\left(w_{2}\right): w_{2} \in\left(w_{1} \lambda_{1}(e) \cap w_{1} \lambda_{2}(e)\right)\right\}, & \text { if } w_{1} \lambda_{1}(e) \cap w_{1} \lambda_{2}(e) \neq \varnothing ; \\ 1 & \text { otherwise; }\end{array}\right.$

for all $w_{1} \in W_{1}$, where $w_{1} \lambda_{1}(e)=\left\{w_{2} \in W_{2}:\left(w_{1}, w_{2}\right) \in \lambda_{1}(e)\right\}$ and $w_{1} \lambda_{2}(e)=\left\{w_{2} \in\right.$ $\left.W_{2}:\left(w_{1}, w_{2}\right) \in \lambda_{2}(e)\right\}$ are called the aftersets of $w_{1}$ for $w_{1} \in W_{1}$ and $e \in A$. Obviously, $\left.\left(\underline{\lambda}_{1}+\lambda_{2}^{T}(e)\right), A\right)$ and $\left(p{\overline{\lambda_{1}+\lambda_{2}}}^{T}(e)\right)$ are two IFS soft sets over $W_{1}$. 
In Definition 12, two soft binary relations from $W_{1}$ to $W_{2}$ are given and an IFS in $W_{2}$ can be approximated as lower and upper approximations with respect to the aftersets. The resulting sets are two pairs of IF soft sets.

Definition 13. Let $W_{1}$ and $W_{2}$ be two non-empty sets, $\left(\lambda_{1}, A\right)$ and $\left(\lambda_{2}, A\right)$ be two soft binary relations from $W_{1}$ to $W_{2}$ and $T=\left\langle\mu_{T}, \gamma_{T}\right\rangle$ be an IFS in $W_{1}$. Then, the pessimistic multigranulation lower approximation ${ }^{T} \underline{\lambda_{1}+\lambda_{2}} p=\left({ }^{\mu_{T}} \underline{\lambda_{1}+\lambda_{2}} p^{\prime}{ }^{\gamma_{T}} \underline{\lambda_{1}+\lambda_{2}} p\right)$ and the pessimistic multigranulation upper approximation ${ }^{T}{\overline{\lambda_{1}+\lambda_{2}}}=\left(\mu_{T}{\overline{\lambda_{1}+\lambda_{2}}}^{p}, \gamma_{T}{\overline{\lambda_{1}+\lambda_{2}}}^{p}\right)$ of $T=\left\langle\mu_{T}, \gamma_{T}\right\rangle$ are IF soft sets over $W_{2}$ and are defined as:

$$
\begin{gathered}
{ }^{\mu_{T}} \underline{\lambda_{1}+\lambda_{2}}(e)\left(w_{2}\right)=\left\{\begin{array}{cc}
\wedge\left\{\mu_{T}\left(w_{1}\right): w_{1} \in\left(\lambda_{1}(e) w_{2} \cap \lambda_{2}(e) w_{2}\right)\right\}, & \text { if } \lambda_{1}(e) w_{2} \cap \lambda_{2}(e) w_{2} \neq \varnothing ; \\
1 & \text { otherwise; }
\end{array}\right. \\
{ }^{\gamma_{T}} \underline{\lambda_{1}+\lambda_{2}} p(e)\left(w_{2}\right)=\left\{\begin{array}{cc}
\vee\left\{\gamma_{T}\left(w_{1}\right): w_{1} \in\left(\lambda_{1}(e) w_{2} \cup \lambda_{2}(e) w_{2}\right)\right\}, & \text { if } \lambda_{1}(e) w_{2} \cup \lambda_{2}(e) w_{2} \neq \varnothing ; \\
0 & \text { otherwise; }
\end{array}\right. \text { and }
\end{gathered}
$$

$$
\begin{aligned}
& \mu_{T}{\overline{\lambda_{1}+\lambda_{2}}}^{p}(e)\left(w_{2}\right)=\left\{\begin{array}{cc}
\vee\left\{\mu_{T}\left(w_{1}\right): w_{1} \in\left(\lambda_{1}(e) w_{2} \cup \lambda_{2}(e) w_{2}\right)\right\}, & \text { if } \lambda_{1}(e) w_{2} \cup \lambda_{2}(e) w_{2} \neq \varnothing ; \\
0 & \text { otherwise; }
\end{array}\right. \\
& \gamma_{T}{\overline{\lambda_{1}+\lambda_{2}}}^{p}(e)\left(w_{2}\right)=\left\{\begin{array}{cc}
\wedge\left\{\gamma_{T}\left(w_{1}\right): w_{1} \in\left(\lambda_{1}(e) w_{2} \cap \lambda_{2}(e) w_{2}\right)\right\}, & \text { if } \lambda_{1}(e) w_{2} \cap \lambda_{2}(e) w_{2} \neq \varnothing ; \\
1 & \text { otherwise; }
\end{array}\right.
\end{aligned}
$$

for all $w_{2} \in W_{2}$ where $\lambda_{1}(e) w_{2}=\left\{w_{1} \in W_{1}:\left(w_{1}, w_{2}\right) \in \lambda_{1}(e)\right\}$ and $\lambda_{2}(e) w_{2}=\left\{w_{1} \in W_{1}:\left(a, w_{2}\right) \in \lambda_{2}(e)\right\}$ are called the foresets of $w_{2}$ for $w_{2} \in W_{2}$ and $e \in A$. Obviously, $\left.\left({ }^{T}{\underline{\lambda_{1}}+\lambda_{2}}_{p}(e)\right), A\right)$ and $\left({ }^{T}{\overline{\lambda_{1}+\lambda_{2}}}^{p}(e)\right)$ are two IFS soft sets over $W_{2}$.

In Definition 13, two soft binary relations from $W_{1}$ to $W_{2}$ are given and an IFS in $W_{1}$ can be approximated as lower and upper approximations with respect to the foresets. The resulting sets are two pairs of IF soft sets.

Of course, $\underline{\lambda_{1}+\lambda_{2}^{T}}{ }_{p}^{T}(e): A \rightarrow I F\left(W_{1}\right), p{\overline{\lambda_{1}+\lambda_{2}}}^{T}(e): A \rightarrow I F\left(W_{1}\right)$ and ${ }^{T} \underline{\lambda_{1}+\lambda_{2}} p(e):$ $A \rightarrow \operatorname{IF}\left(W_{2}\right),{ }^{T}{\overline{\lambda_{1}+\lambda_{2}}}^{p}(e): A \rightarrow I F\left(W_{2}\right)$.

The following example explains the above definitions.

Example 1. Let $W_{1}=\{a, b, c, d, e, f\}, W_{2}=\{x, y, z\}$ and $A=\left\{e_{1}, e_{2}, e_{3}\right\}$, and $\left(\lambda_{1}, A\right)$ and $\left(\lambda_{2}, A\right)$ be two soft binary relations from $W_{1}$ to $W_{2}$ defined by

$$
\begin{aligned}
& \lambda_{1}\left(e_{1}\right)=\{(a, x),(a, y),(b, z),(c, x),(c, z),(d, x),(d, y),(d, z),(f, x),(f, y)\}, \\
& \lambda_{1}\left(e_{2}\right)=\{(a, z),(b, x),(b, y),(b, z),(c, x),(c, y),(e, y),(e, z),(f, x)\}, \\
& \lambda_{1}\left(e_{3}\right)=\{(c, y),(c, z),(d, x),(d, y),(e, z),(f, z)\} \\
& \lambda_{2}\left(e_{1}\right)=\{(b, x),(c, y),(d, x),(d, z),(e, z)\}, \\
& \lambda_{2}\left(e_{2}\right)=\{(a, y),(a, z),(c, x),(c, y),(c, z),(e, z),(f, z)\} \text { and } \\
& \lambda_{2}\left(e_{3}\right)=\{(a, x),(a, y),(a, z),(b, y),(f, y)\} .
\end{aligned}
$$


Then, their aftersets and foresets are

$$
\begin{aligned}
& a \lambda_{1}\left(e_{1}\right)=\{x, y\}, b \lambda_{1}\left(e_{1}\right)=\{z\}, \quad c \lambda_{1}\left(e_{1}\right)=\{x, z\}, d \lambda_{1}\left(e_{1}\right)=\{x, y, z\}, \\
& e \lambda_{1}\left(e_{1}\right)=\varnothing, \quad f \lambda_{1}\left(e_{1}\right)=\{x, y\}, \\
& a \lambda_{1}\left(e_{2}\right)=\{z\}, b \lambda_{1}\left(e_{2}\right)=\{x, y, z\}, c \lambda_{1}\left(e_{2}\right)=\{x, y\}, d \lambda_{1}\left(e_{2}\right)=\varnothing, \\
& e \lambda_{1}\left(e_{2}\right)=\{y, z\}, f \lambda_{1}\left(e_{2}\right)=\{x\} \text { and } \\
& a \lambda_{1}\left(e_{3}\right)=\varnothing, \quad b \lambda_{1}\left(e_{3}\right)=\varnothing, \quad c \lambda_{1}\left(e_{3}\right)=\{y, z\}, d \lambda_{1}\left(e_{3}\right)=\{x, y\}, \\
& e \lambda_{1}\left(e_{3}\right)=\{z\}, f \lambda_{1}\left(e_{3}\right)=\{z\} \text {. } \\
& a \lambda_{2}\left(e_{1}\right)=\varnothing, \quad b \lambda_{2}\left(e_{1}\right)=\{x\}, \quad c \lambda_{2}\left(e_{1}\right)=\{y\}, \quad d \lambda_{2}\left(e_{1}\right)=\{x, z\}, \\
& e \lambda_{2}\left(e_{1}\right)=\{z\}, f \lambda_{2}\left(e_{1}\right)=\varnothing, \\
& a \lambda_{2}\left(e_{2}\right)=\{y, z\}, b \lambda_{2}\left(e_{2}\right)=\varnothing, \quad c \lambda_{2}\left(e_{2}\right)=\{x, y, z\}, d \lambda_{2}\left(e_{2}\right)=\varnothing \text {, } \\
& e \lambda_{2}\left(e_{2}\right)=\{z\}, \quad f \lambda_{2}\left(e_{2}\right)=\{z\} \text { and } \\
& a \lambda_{2}\left(e_{3}\right)=\{x, y, z\}, b \lambda_{2}\left(e_{3}\right)=\{y\}, \quad c \lambda_{2}\left(e_{3}\right)=\varnothing, \quad d \lambda_{2}\left(e_{3}\right)=\varnothing, \\
& e \lambda_{2}\left(e_{3}\right)=\varnothing, \quad f \lambda_{2}\left(e_{3}\right)=\{y\} \text {. } \\
& \lambda_{1}\left(e_{1}\right) x=\{a, c, d, f\}, \lambda_{1}\left(e_{1}\right) y=\{a, d, f\}, \quad \lambda_{1}\left(e_{1}\right) z=\{b, c, d\}, \\
& \lambda_{1}\left(e_{2}\right) x=\{b, c, f\}, \quad \lambda_{1}\left(e_{2}\right) y=\{b, c, e\}, \quad \lambda_{1}\left(e_{2}\right) z=\{a, b, e\} \text { and } \\
& \lambda_{1}\left(e_{3}\right) x=\{d\}, \quad \lambda_{1}\left(e_{3}\right) y=\{c, d\}, \quad \lambda_{1}\left(e_{3}\right) z=\{c, e, f\} . \\
& \lambda_{2}\left(e_{1}\right) x=\{b, d\}, \quad \lambda_{2}\left(e_{1}\right) y=\{c\}, \quad \lambda_{2}\left(e_{1}\right) z=\{d, e\}, \\
& \lambda_{2}\left(e_{2}\right) x=\{c\}, \quad \lambda_{2}\left(e_{2}\right) y=\{a, c\}, \quad \lambda_{2}\left(e_{2}\right) z=\{a, c, e, f\} \text { and } \\
& \lambda_{2}\left(e_{3}\right) x=\{a\}, \quad \lambda_{2}\left(e_{3}\right) y=\{a, b, f\}, \quad \lambda_{2}\left(e_{3}\right) z=\{a\} \text {. }
\end{aligned}
$$

\begin{tabular}{|c|c|c|c|c|c|c|}
\hline & $a$ & $b$ & $c$ & $d$ & $e$ & $f$ \\
\hline$\left({\underline{\lambda_{1}+\lambda_{2}}}_{p}^{\mu_{T_{1}}}\left(e_{1}\right),{\underline{\lambda_{1}+\lambda_{2}}}_{p}^{\gamma_{T_{1}}}\left(e_{1}\right)\right)$ & $(1,0.4)$ & $(1,0.9)$ & $(1,0.9)$ & $(0.1,0.9)$ & $(1,0.9)$ & $(1,0.4)$ \\
\hline$\left(\underline{\lambda}_{1}+\lambda_{2}{ }_{p}^{\mu_{T_{1}}}\left(e_{2}\right), \underline{\lambda_{1}+\lambda_{2}} \underset{p}{\gamma_{T_{1}}}\left(e_{2}\right)\right)$ & $(0.1,0.9)$ & $(1,0.9)$ & $(0.5,0.9)$ & $(1,0.0)$ & $(0.1,0.9)$ & $(1,0.9)$ \\
\hline$\left({\underline{\lambda_{1}}+\lambda_{2}}_{p}^{\mu_{T_{1}}}\left(e_{3}\right),{\underline{\lambda_{1}}+\lambda_{2}}_{p}^{\gamma_{T_{1}}}\left(e_{3}\right)\right)$ & $(1,0.9)$ & $(1,0.4)$ & $(1,0.9)$ & $(1,0.4)$ & $(1,0.9)$ & $(1,0.9)$ \\
\hline$\left(p{\overline{\lambda_{1}+\lambda_{2}}}^{\mu_{T_{1}}}\left(e_{1}\right), p{\overline{\lambda_{1}+\lambda_{2}}}^{\gamma}{ }_{T_{1}}\left(e_{1}\right)\right)$ & $(0.6,1)$ & $(0.6,1)$ & $(0.6,1)$ & $(0.6,0.1)$ & $(0.1,1)$ & $(0.6,1)$ \\
\hline$\left(p{\overline{\lambda_{1}+\lambda_{2}}}^{\mu_{T_{1}}}\left(e_{2}\right), p \overline{\lambda_{1}+\lambda_{2}} \gamma_{T_{1}}\left(e_{2}\right)\right)$ & $(0.5,0.9)$ & $(0.6,1)$ & $(0.6,0.3)$ & $(0.0,1)$ & $(0.5,0.9)$ & $(0.6,1)$ \\
\hline$\left(p{\overline{\lambda_{1}+\sigma_{2}}}^{\mu_{T_{1}}}\left(e_{3}\right), p{\overline{\lambda_{1}+\lambda_{2}}}^{\gamma_{T_{1}}}\left(e_{3}\right)\right)$ & $(0.6,1)$ & $(0.5,1)$ & $(0.5,1)$ & $(0.6,1)$ & $(0.1,1)$ & $(0.5,1)$ \\
\hline
\end{tabular}

(1) Define $T_{1}=\left\langle\mu_{T_{1}}, \gamma_{T_{1}}\right\rangle: W_{2} \rightarrow[0,1]$ as given in Table 1:

Table 1. Intuitionistic fuzzy set $T_{1}$.

\begin{tabular}{llll}
\hline $\boldsymbol{T}_{\mathbf{1}}$ & $\boldsymbol{x}$ & $\boldsymbol{y}$ & $\boldsymbol{z}$ \\
\hline$\left(\mu_{T_{1}}, \gamma_{T_{1}}\right)$ & $(0.6,0.3)$ & $(0.5,0.4)$ & $(0.1,0.9)$ \\
\hline
\end{tabular}

The pessimistic multigranulation lower and upper approximations of $T_{1}$ with respect to the aftersets are given in Table 2.

Table 2. Pessimistic multigranulation lower and upper approximations of $T_{1}$.

(2) Define $T_{2}=\left\langle\mu_{T_{2}}, \gamma_{T_{2}}\right\rangle: T_{1} \rightarrow[0,1]$ as given in Table 3:

Table 3. Intuitionistic fuzzy set $T_{2}$.

\begin{tabular}{lllllll}
\hline $\boldsymbol{T}_{\mathbf{2}}$ & $\boldsymbol{a}$ & $\boldsymbol{b}$ & $\boldsymbol{c}$ & $\boldsymbol{d}$ & $\boldsymbol{e}$ & $\boldsymbol{f}$ \\
\hline$\left(\mu_{\mathrm{T}_{2}}, \gamma_{T_{2}}\right)$ & $(0.9,0.0)$ & $(0.6,0.3)$ & $(0.7,0.3)$ & $(0.5,0.4)$ & $(0.3,0.7)$ & $(0.1,0.7)$ \\
\hline
\end{tabular}


The pessimistic multigranulation lower and upper approximations of $T_{2}$ with respect to the foresets are given in Table 4 .

Table 4. Pessimistic multigranulation lower and upper approximations of $T_{2}$.

\begin{tabular}{|c|c|c|c|}
\hline & $x$ & $y$ & $z$ \\
\hline$\left({ }^{\mu_{T_{2}}} \underline{\lambda_{1}+\lambda_{2}} p\left(e_{1}\right),{ }^{\gamma_{T_{2}}} \underline{\lambda_{1}+\lambda_{2}} p\left(e_{1}\right)\right)$ & $(0.5,0.7)$ & $(1,0.7)$ & $(0.5,0.7)$ \\
\hline$\left({ }^{\mu_{T_{2}}} \underline{\lambda_{1}+\lambda_{2}} p\left(e_{2}\right),{ }^{\gamma T_{2}} \underline{\lambda_{1}+\lambda_{2}} p\left(e_{2}\right)\right)$ & $(0.7,0.7)$ & $(0.7,0.7)$ & $(0.3,0.7)$ \\
\hline$\left({ }^{\mu_{T_{2}}} \underline{\lambda_{1}+\lambda_{2}} p\left(e_{3}\right),{ }^{\gamma_{T_{2}}} \underline{\lambda_{1}+\lambda_{2}} p\left(e_{3}\right)\right)$ & $(1,0.4)$ & $(1,0.7)$ & $(1,0.7)$ \\
\hline 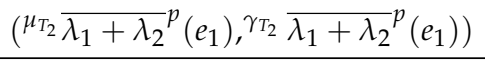 & $(0.9,0.4)$ & $(0.9,1)$ & $(0.7,0.4)$ \\
\hline$\left(\mu_{T_{2}}{\overline{\lambda_{1}+\lambda_{2}}}^{p}\left(e_{2}\right), \gamma_{T_{2}}{\overline{\lambda_{1}+\lambda_{2}}}^{p}\left(e_{2}\right)\right)$ & $(0.7,0.3)$ & $(0.9,0.3)$ & $(0.9,0.0)$ \\
\hline 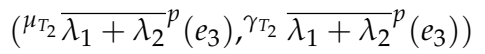 & $(0.9,1)$ & $(0.9,1)$ & $(0.9,1)$ \\
\hline
\end{tabular}

Table 2 shows the pessimistic multigranulation lower and upper approximations of IFS $T_{1}$ with respect to the aftersets by using Definition 12 . Table 4 shows the pessimistic multigranulation lower and upper approximations of IFS $T_{2}$ with respect to the foresets by using Definition 13.

Proposition 1. Let $\left(\lambda_{1}, A\right),\left(\lambda_{2}, A\right)$ be two soft relations from $W_{1}$ to $W_{2}$; that is, $\lambda_{1}: A \rightarrow$ $P\left(W_{1} \times W_{2}\right)$ and $\lambda_{2}: A \rightarrow P\left(W_{1} \times W_{2}\right)$ and $T \in I F\left(W_{2}\right)$. Then, the following hold with respect to the after sets.

(1) ${\underline{\lambda_{1}}+\lambda_{2}^{T}}_{p}^{T} \geqslant{\underline{\lambda_{1}}}^{T} \wedge{\underline{\lambda_{2}}}^{T}$;

(2) $p{\overline{\lambda_{1}+\lambda_{2}}}^{T}={\overline{\lambda_{1}}}^{T} \vee{\overline{\lambda_{2}}}^{T}$.

Proof. (1) Let $w_{1} \in W_{1}$. Then, ${\underline{\lambda_{1}}+\lambda_{2}{ }_{p}^{\mu}}_{p}(e)\left(w_{1}\right)=\wedge\left\{\mu_{T}\left(w_{2}\right): w_{2} \in\left(w_{1} \lambda_{1}(e) \cap w_{1} \lambda_{2}(e)\right)\right\}$ $\geqslant\left(\wedge\left\{\mu_{T}\left(w_{2}\right): w_{2} \in\left(w_{1} \lambda_{1}(e)\right\}\right) \wedge\left(\wedge\left\{\mu_{T}\left(w_{2}\right): w_{2} \in\left(w_{1} \lambda_{2}(e)\right\}\right)={\overline{\lambda_{1}}}^{\mu_{T}}(e)\left(w_{1}\right) \wedge\right.\right.$ ${\overline{\lambda_{2}}}^{\mu_{T}}(e)\left(w_{1}\right)$.

Similarly, let $w_{1} \in W_{1}$. Then, $\underline{\lambda}_{1}+\lambda_{2}^{\gamma_{T}}(e)\left(w_{1}\right)=\vee\left\{\gamma_{T}\left(w_{2}\right): w_{2} \in\left(w_{1} \lambda_{1}(e) \cup\right.\right.$ $\left.\left.w_{1} \lambda_{2}(e)\right)\right\}=\left(\vee\left\{\gamma_{T}\left(w_{2}\right): w_{2} \in\left(w_{1} \lambda_{1}(e)\right\}\right) \vee\left(\vee\left\{\gamma_{T}\left(w_{2}\right): w_{2} \in\left(w_{1} \lambda_{2}(e)\right\}\right)=\bar{\lambda}_{1} \gamma_{T}(e)\right.\right.$ $\left(w_{1}\right) \vee \underline{\lambda}_{2}{ }^{\gamma_{T}}(e)\left(w_{1}\right)$.

Hence, $\underline{\lambda_{1}+\lambda_{2}^{T}} p={\underline{\lambda_{1}}}^{T} \wedge{\underline{\lambda_{2}}}^{T}$.

(2) Let $w_{1} \in W_{1}$. Then, $p{\overline{\lambda_{1}+\lambda_{2}}}^{\mu_{T}}(e)\left(w_{1}\right)=\vee\left\{\mu_{T}\left(w_{2}\right): w_{2} \in\left(w_{1} \lambda_{1}(e) \cup w_{1} \lambda_{2}(e)\right)\right\}=$ $\left(\vee\left\{\mu_{T}\left(w_{2}\right): w_{2} \in\left(w_{1} \lambda_{1}(e)\right\}\right) \vee\left(\vee\left\{\mu_{T}\left(w_{2}\right): w_{2} \in\left(w_{1} \lambda_{2}(e)\right\}\right)=\overline{\lambda_{1}} \mu_{T}(e)\left(w_{1}\right) \vee \frac{\lambda_{2}}{\mu_{T}}\right.\right.$ (e) $\left(w_{1}\right)$.

Similarly, let $w_{1} \in W_{1}$. Then, $p{\overline{\lambda_{1}+\lambda_{2}}}^{\gamma}(e)\left(w_{1}\right)=\wedge\left\{\gamma_{T}\left(w_{2}\right): w_{2} \in\left(w_{1} \lambda_{1}(e) \cap\right.\right.$ $\left.\left.w_{1} \lambda_{2}(e)\right)\right\} \geqslant\left(\wedge\left\{\gamma_{T}\left(w_{2}\right): w_{2} \in\left(w_{1} \lambda_{1}(e)\right\}\right) \wedge\left(\wedge\left\{\gamma_{T}\left(w_{2}\right): w_{2} \in\left(w_{1} \lambda_{2}(e)\right\}\right)=\overline{\lambda_{1}} \gamma_{T}\right.\right.$ $(e)\left(w_{1}\right) \wedge \bar{\lambda}_{2}^{\gamma_{T}}(e)\left(w_{1}\right)$

Hence, $p{\overline{\lambda_{1}+\lambda_{2}}}^{T}={\overline{\lambda_{1}}}^{T} \vee{\overline{\lambda_{2}}}^{T}$.

For the converse, we have the following example.

Example 2. (Continued from Example 1). According to Example 1, we have the following: 


$$
\begin{aligned}
& \lambda_{1}{ }^{\mu_{T}}\left(e_{1}\right)(c)=0.1 \text { and }{\underline{\lambda_{2}}}^{\mu_{T}}\left(e_{1}\right)(c)=0.5 ; \\
& \lambda_{1}{ }^{\gamma_{T}}\left(e_{1}\right)(c)=0.9 \text { and } \underline{\lambda}_{2}{ }^{\gamma_{T}}\left(e_{1}\right)(c)=0.4 \text {; } \\
& {\underline{\lambda_{1}}}^{\mu_{T}}\left(e_{2}\right)(c)=0.5 \text { and }{\underline{\lambda_{2}}}^{\mu_{T}}\left(e_{2}\right)(c)=0.1 \text {; } \\
& {\underline{\lambda_{1}}}^{\gamma_{T}}\left(e_{2}\right)(c)=0.4 \text { and }{\underline{\lambda_{2}}}^{\gamma_{T}}\left(e_{2}\right)(c)=0.9 \text {; } \\
& {\underline{\lambda_{1}}}^{\mu_{T}}\left(e_{3}\right)(c)=0.1 \text { and }{\underline{\lambda_{2}}}^{\mu_{T}}\left(e_{3}\right)(c)=1.0 \text {; } \\
& \lambda_{1}{ }^{\gamma_{T}}\left(e_{3}\right)(c)=0.9 \text { and }{\underline{\lambda_{2}}}^{\gamma_{T}}\left(e_{3}\right)(c)=0.0 \text {; } \\
& {\overline{\lambda_{1}}}^{\mu_{T}}\left(e_{3}\right)(b)=0.0 \text { and }{\overline{\lambda_{2}}}^{\mu_{T}}\left(e_{3}\right)(b)=0.5 \text {; } \\
& {\overline{\lambda_{1}}}^{\gamma}\left(e_{3}\right)(b)=0.0 \text { and }{\overline{\lambda_{2}}}^{\gamma_{T}}\left(e_{3}\right)(b)=0.4 \text {. }
\end{aligned}
$$

Hence,

$$
\begin{aligned}
& {\underline{\lambda_{1}}+\lambda_{2}}_{p}^{\mu_{T}}\left(e_{1}\right)(c)=1.0 \nless 0.1=\underline{\lambda}_{1}^{\mu_{T}}\left(e_{1}\right)(c) \wedge \underline{\lambda}^{\mu_{T}}\left(e_{1}\right)(c) ; \\
& {\underline{\lambda_{1}+\lambda_{2}}}_{p}^{\gamma_{T}}\left(e_{1}\right)(c)=0.9=0.9={\underline{\lambda_{1}}}^{\gamma_{T}}\left(e_{1}\right)(c) \vee{\underline{\lambda_{2}}}^{\gamma_{T}}\left(e_{1}\right)(c) ; \\
& {\underline{\lambda_{1}}+\lambda_{2}}_{p}^{\mu_{T}}\left(e_{2}\right)(c)=0.5 \nless 0.1=\underline{\lambda}_{1}^{\mu_{T}}\left(e_{2}\right)(c) \wedge{\underline{\lambda_{2}}}^{\mu_{T}}\left(e_{2}\right)(c) ; \\
& {\underline{\lambda_{1}}+\lambda_{2} \gamma_{p}}_{\gamma_{T}}\left(e_{2}\right)(c)=0.9=0.9={\underline{\lambda_{1}}}^{\gamma_{T}}\left(e_{2}\right)(c) \vee{\underline{\lambda_{2}}}^{\gamma_{T}}\left(e_{2}\right)(c) ; \\
& {\underline{\lambda_{1}+\lambda_{2}}}_{0}^{\mu_{T}}\left(e_{3}\right)(c)=1.0 \nless 0.1={\underline{\lambda_{1}}}^{\mu_{T}}\left(e_{3}\right)(c) \wedge \underline{\lambda}_{2}^{\mu_{T}}\left(e_{3}\right)(c) ; \\
& {\underline{\lambda_{1}+\lambda_{2}}}_{p}^{\gamma_{T}}\left(e_{3}\right)(c)=0.9=0.9={\underline{\lambda_{1}}}^{\gamma_{T}}\left(e_{3}\right)(c) \vee{\underline{\lambda_{2}}}^{\gamma_{T}}\left(e_{3}\right)(c) ; \\
& p{\overline{\lambda_{1}+\lambda_{2}}}^{\mu_{T}}\left(e_{3}\right)(b)=0.5=0.5={\overline{\lambda_{1}}}^{\mu_{T}}\left(e_{3}\right)(b) \vee{\overline{\lambda_{2}}}^{\mu_{T}}\left(e_{3}\right)(b) ; \\
& p{\overline{\lambda_{1}+\lambda_{2}}}^{\gamma_{T}}\left(e_{3}\right)(b)=1.0 \nless 0.4={\overline{\lambda_{1}}}^{\gamma_{T}}\left(e_{3}\right)(b) \vee{\overline{\lambda_{2}}}^{\gamma_{T}}\left(e_{3}\right)(b) \text {. }
\end{aligned}
$$

Proposition 2. Let $\left(\lambda_{1}, A\right),\left(\lambda_{2}, A\right)$ be two soft relations from $W_{1}$ to $W_{2}$; that is, $\lambda_{1}: A \rightarrow$ $P\left(W_{1} \times W_{2}\right)$ and $\lambda_{2}: A \rightarrow P\left(W_{1} \times W_{2}\right)$ and $T \in I F\left(W_{1}\right)$. Then, the following hold with respect to the foresets.

(1) ${ }^{T} \underline{\lambda_{1}+\lambda_{2}} p \geqslant{ }^{T} \underline{\lambda_{1}} \wedge^{T} \underline{\lambda_{2}}$

(2) $T{\overline{\bar{\lambda}_{1}+\lambda_{2}}}^{p}={ }^{T} \overline{\overline{\lambda_{1}}} \vee^{T} \overline{\lambda_{2}}$.

Proof. The proof is similar to the proof of Proposition 1.

Proposition 3. Let $\left(\lambda_{1}, A\right),\left(\lambda_{2}, A\right)$ be two soft relations from $W_{1}$ to $W_{2}$; that is, $\lambda_{1}: A \rightarrow$ $P\left(W_{1} \times W_{2}\right)$ and $\lambda_{2}: A \rightarrow P\left(W_{1} \times W_{2}\right)$ and $T \in I F\left(W_{2}\right)$. Then, the following hold.

(1) $\underline{\lambda}_{1}+\lambda_{2}{ }_{p}^{W_{2}}=1_{W_{1}}$ for all $e \in A$;

(2) $p{\overline{\lambda_{1}+\lambda_{2}}}^{1} W_{2}=1_{W_{1}}$ if $w_{1} \lambda_{1}(e) \cup w_{1} \lambda_{2}(e) \neq \varnothing$ and $w_{1} \lambda_{1}(e) \cap w_{1} \lambda_{2}(e) \neq \varnothing$;

(3) $\underline{\lambda}_{1}+\lambda_{2}^{0}{ }_{W_{2}}=0_{W_{1}}$ if $w_{1} \lambda_{1}(e) \cap w_{1} \lambda_{2}(e) \neq \varnothing$ and $w_{1} \lambda_{1}(e) \cup w_{1} \lambda_{2}(e) \neq \varnothing$;

(4) $p{\overline{\lambda_{1}+\lambda_{2}}}^{0}{ }_{W_{2}}=0_{W_{1}}$ for all $e \in A$.

Proof. (1) Let $w_{1} \in W_{1}$ and $1_{W_{2}}=\langle 1,0\rangle$ be the universal set of $W_{2}$. If $w_{1} \lambda_{1}(e) \cap w_{1} \lambda_{2}(e)=$ $\varnothing$, then $\underline{\lambda_{1}+\lambda_{2}^{1}} p(e)\left(w_{1}\right)=1$ and $\underline{\lambda_{1}+\lambda_{2}^{0}} p(e)\left(w_{1}\right)=0$.

If $w_{1} \lambda_{1}(e) \cap w_{1} \lambda_{2}(e) \neq \varnothing$, then $\underline{\lambda_{1}+\lambda_{2}^{1}} p(e)\left(w_{1}\right)=\wedge\left\{1\left(w_{2}\right): w_{2} \in\left(w_{1} \lambda_{1}(e) \cap\right.\right.$ $\left.\left.w_{1} \lambda_{2}(e)\right)\right\}=\wedge\left\{1: w_{2} \in\left(w_{1} \lambda_{1}(e) \cap w_{1} \lambda_{2}(e)\right)\right\}=1$,

and $\underline{\lambda_{1}+\lambda_{2}^{0}} p(e)\left(w_{1}\right)=\vee\left\{0\left(w_{2}\right): w_{2} \in\left(w_{1} \lambda_{1}(e) \cup w_{1} \lambda_{2}(e)\right)\right\}=\vee\left\{0: w_{2} \in\left(w_{1} \lambda_{1}(e)\right.\right.$ $\left.\left.\cup w_{1} \lambda_{2}(e)\right)\right\}=0$.

(2) Let $w_{1} \in W_{1}$ and $1_{W_{2}}=\langle 1,0\rangle$ be the universal set of $W_{2}$. If $w_{1} \lambda_{1}(e) \cup w_{1} \lambda_{2}(e) \neq \varnothing$, then ${\overline{\lambda_{1}+\lambda_{2}}}^{1}(e)\left(w_{1}\right)=\vee\left\{1\left(w_{2}\right): w_{2} \in\left(w_{1} \lambda_{1}(e) \cup w_{1} \lambda_{2}(e)\right)\right\}=\vee\left\{1: w_{2} \in\left(w_{1} \lambda_{1}(e) \cup\right.\right.$ $\left.\left.w_{1} \lambda_{2}(e)\right)\right\}=1$,

and $p \overline{\lambda_{1}+\lambda_{2}}(e)\left(w_{1}\right)=\wedge\left\{0\left(w_{2}\right): w_{2} \in\left(w_{1} \lambda_{1}(e) \cap w_{1} \lambda_{2}(e)\right)\right\}=\wedge\left\{0: w_{2} \in\right.$ $\left.\left(w_{1} \lambda_{1}(e) \cap w_{1} \lambda_{2}(e)\right)\right\}=0$. 
(3) Let $w_{1} \in W_{1}$ and $0_{W_{2}}=\langle 0,1\rangle$ be the universal set of $W_{2}$. If $w_{1} \lambda_{1}(e) \cap w_{1} \lambda_{2}(e) \neq \varnothing$, then $\underline{\lambda}_{1}+\lambda_{2}^{0}(e)\left(w_{1}\right)=\wedge\left\{0\left(w_{2}\right): w_{2} \in\left(w_{1} \lambda_{1}(e) \cap w_{1} \lambda_{2}(e)\right)\right\}=\wedge\left\{0: w_{2} \in\left(w_{1} \lambda_{1}(e) \cap\right.\right.$ $\left.\left.w_{1} \lambda_{2}(e)\right)\right\}=0$, and $\underline{\lambda_{1}+\lambda_{2}^{1}} p(e)\left(w_{1}\right)=\vee\left\{1\left(w_{2}\right): w_{2} \in\left(w_{1} \lambda_{1}(e) \cup w_{1} \lambda_{2}(e)\right)\right\}=\vee\left\{1: w_{2} \in\left(w_{1} \lambda_{1}(e)\right.\right.$ $\left.\left.\cup w_{1} \lambda_{2}(e)\right)\right\}=1$.

(4) The properties can be proved similarly to (3).

Proposition 4. Let $\left(\lambda_{1}, A\right),\left(\lambda_{2}, A\right)$ be two soft relations from $W_{1}$ to $W_{2}$; that is, $\lambda_{1}: A \rightarrow$ $P\left(W_{1} \times W_{2}\right)$ and $\lambda_{2}: A \rightarrow P\left(W_{1} \times W_{2}\right)$ and $T \in I F\left(W_{1}\right)$. Then, the following holds.

(1) ${ }^{W_{1}} \underline{\lambda_{1}+\lambda_{2}} p=1_{W_{2}}$ for all $e \in A$;

(2) $1_{W_{1}}{\overline{\lambda_{1}+\lambda_{2}}}^{p}=1_{W_{2}}$ for all $e \in A$, if $\lambda_{1}(e) w_{2} \cup \lambda_{2}(e) w_{2} \neq \varnothing$ and $\lambda_{1}(e) w_{2} \cap \lambda_{2}(e) w_{2} \neq \varnothing$;

(3) ${ }^{0} W_{1} \underline{\lambda_{1}+\lambda_{2}} p=0_{W_{2}}$ for all $e \in A$, if $\lambda_{1}(e) w_{2} \cap \lambda_{2}(e) w_{2} \neq \varnothing$ and $\lambda_{1}(e) w_{2} \cup \lambda_{2}(e) w_{2} \neq \varnothing$;

(4) $0_{W_{1}}{\overline{\lambda_{1}+\lambda_{2}}}^{p}$ for all $e \in A$.

Proof. The proof is similar to the proof of Proposition 3.

Proposition 5. Let $\left(\lambda_{1}, A\right),\left(\lambda_{2}, A\right)$ be two soft relations from $W_{1}$ to $W_{2}$; that is, $\lambda_{1}: A \rightarrow$ $P\left(W_{1} \times W_{2}\right)$ and $\lambda_{2}: A \rightarrow P\left(W_{1} \times W_{2}\right)$ and $T, T_{1}, T_{2} \in \operatorname{IF}\left(W_{2}\right)$. Then, the following properties hold.

(1) If $T_{1} \subseteq T_{2}$, then $\underline{\lambda_{1}+\lambda_{2}^{T_{1}}} \subseteq \underline{\lambda_{1}+\lambda_{2}^{2}} p$;

(2) If $T_{1} \subseteq T_{2}$, then $p{\overline{\lambda_{1}+\lambda_{2}}}^{T} \subseteq p{\overline{\lambda_{1}+\lambda_{2}}}^{T}$;

(3) $\quad{\underline{\lambda_{1}}+\lambda_{2}}_{p}^{T_{1} \cap T_{2}}=\underline{\lambda_{1}+\lambda_{2}^{T_{1}}} \cap \underline{\lambda_{1}+\lambda_{2}^{T_{2}}}$;

(4) $\quad{\underline{\lambda_{1}+\lambda_{2}}}_{p}^{T_{1} \cup T_{2}} \supseteq \underline{\lambda_{1}+\lambda_{2}^{T_{1}}} \cup \underline{\lambda_{1}+\lambda_{2}^{T_{2}}} p$;

(5) $p{\overline{\lambda_{1}+\lambda_{2}}}^{T} \cup T_{2}=p{\overline{\lambda_{1}+\lambda_{2}}}^{T} \cup p{\overline{\lambda_{1}+\lambda_{2}}}^{T}$;

(6) $p{\overline{\lambda_{1}+\lambda_{2}}}^{T} T_{1} T_{2} \subseteq p{\overline{\lambda_{1}+\lambda_{2}}}^{T} \cap^{p}{\overline{\lambda_{1}+\lambda_{2}}}^{T}$.

Proof. (1) Since $T_{1} \subseteq T_{2}, \mu_{T_{1}} \leqslant \mu_{T_{2}}$ and $\gamma_{T_{1}} \geqslant \gamma_{T_{2}}$. Thus, $\underline{\lambda}_{1}+\lambda_{2} \mu_{p} T_{1}(e)\left(w_{1}\right)=\wedge\left\{\mu_{T_{1}}\left(w_{2}\right)\right.$ : $\left.w_{2} \in\left(w_{1} \lambda_{1}(e) \cap w_{1} \lambda_{2}(e)\right)\right\} \leqslant \wedge\left\{\mu_{T_{2}}\left(w_{2}\right): w_{2} \in\left(w_{1} \lambda_{1}(e) \cap w_{1} \lambda_{2}(e)\right)\right\}=\underline{\lambda_{1}+\lambda_{2}}{ }_{p}^{\mu_{T}}$ $(e)\left(w_{1}\right)$,

and $\underline{\lambda_{1}+\lambda_{2}}{ }_{p}^{\gamma_{T_{1}}}(e)\left(w_{1}\right)=\vee\left\{\gamma_{T_{1}}\left(w_{2}\right): w_{2} \in\left(w_{1} \lambda_{1}(e) \cup u w_{1} \lambda_{2}(e)\right)\right\} \geqslant \vee\left\{\gamma_{T_{2}}\left(w_{2}\right):\right.$ $\left.w_{2} \in\left(w_{1} \lambda_{1}(e) \cup w_{1} \lambda_{2}(e)\right)\right\}=\underline{\lambda_{1}+\lambda_{2}}{ }_{p}^{\gamma_{T}}(e)\left(w_{1}\right)$.

(2) This can be proved similar to (1).

(3) Let $w_{1} \in W_{1}$. If $w_{1} \lambda_{1}(e) \cap w_{1} \lambda_{2}(e)=\varnothing$, then $\underline{\lambda}_{1}+\lambda_{2}^{\mu_{T_{1}} \cap T_{2}}(e)\left(w_{1}\right)=1=$ $\underline{\lambda_{1}+\lambda_{2}^{2}} \mu_{T_{1}}^{\mu_{1}}(e)\left(w_{1}\right) \cap \underline{\lambda_{1}+\lambda_{2}}{ }_{0}^{\mu_{T}}(e)\left(w_{1}\right)$

and $\underline{\lambda_{1}+\lambda_{2}} \underset{p}{\gamma_{T_{1} \cap T_{2}}}(e)\left(w_{1}\right)=0=\underline{\lambda_{1}+\lambda_{2}} \underset{p}{\gamma_{T_{1}}}(e)\left(w_{1}\right) \cup \underline{\lambda_{1}+\lambda_{2}^{\gamma}}{ }_{p}^{\gamma T_{2}}(e)\left(w_{1}\right)$.

If $w_{1} \lambda_{1}(e) \cap w_{1} \lambda_{2}(e) \neq \varnothing$,

then $\underline{\lambda}_{1}+\lambda_{2}^{\mu_{T_{1}} \cap T_{2}}(e)\left(w_{1}\right)=\wedge\left\{\left(\mu_{T_{1}} \wedge \mu_{T_{2}}\right)\left(w_{2}\right): w_{2} \in\left(w_{1} \lambda_{1}(e) \cap w_{1} \lambda_{2}(e)\right)\right\}=$ $\wedge\left\{\mu_{T_{1}}\left(w_{2}\right) \wedge \mu_{T_{2}}\left(w_{2}\right): w_{2} \in\left(w_{1} \lambda_{1}(e) \cap w_{1} \lambda_{2}(e)\right)\right\}$

$=\left(\wedge\left\{\mu_{T_{1}}\left(w_{2}\right): w_{2} \in\left(w_{1} \lambda_{1}(e) \cap w_{1} \lambda_{2}(e)\right)\right\}\right) \wedge\left(\wedge\left\{\mu_{T_{2}}\left(w_{2}\right): w_{2} \in\left(w_{1} \lambda_{1}(e) \cap\right.\right.\right.$ $\left.\left.\left.w_{1} \lambda_{2}(e)\right)\right\}\right)$

$={\underline{\lambda_{1}}+\lambda_{2}}_{p}^{\mu_{T_{1}}}(e)\left(w_{1}\right) \cap{\underline{\lambda_{1}}+\lambda_{2}^{2}}_{p}^{\mu_{T_{2}}}(e)\left(w_{1}\right)$.

In addition, $\lambda_{1}+\lambda_{2}^{\gamma_{T_{1}} \cap T_{2}}(e)\left(w_{1}\right)=\vee\left\{\left(\mu_{T_{1}} \vee \mu_{T_{2}}\right)\left(w_{2}\right): w_{2} \in\left(w_{1} \lambda_{1}(e) \cup w_{1} \lambda_{2}(e)\right)\right\}=$ $\vee\left\{\mu_{T_{1}}\left(w_{2}\right) \vee \mu_{T_{2}}\left(w_{2}\right): w_{2} \in\left(w_{1} \lambda_{1}(e) \cup w_{1} \lambda_{2}(e)\right)\right\}$

$=\left(\vee\left\{\mu_{T_{1}}\left(w_{2}\right): w_{2} \in\left(w_{1} \lambda_{1}(e) \cup w_{1} \lambda_{2}(e)\right)\right\}\right) \vee\left(\vee\left\{\mu_{T_{2}}\left(w_{2}\right): w_{2} \in\left(w_{1} \lambda_{1}(e) \cup\right.\right.\right.$ $\left.\left.\left.w_{1} \lambda_{2}(e)\right)\right\}\right)$

$=\underline{\lambda_{1}+\lambda_{2}}{ }_{p}^{\mu_{T_{1}}}(e)\left(w_{1}\right) \cup \underline{\lambda_{1}+\lambda_{2}}{ }_{p}^{\mu_{T_{2}}}(e)\left(w_{1}\right)$.

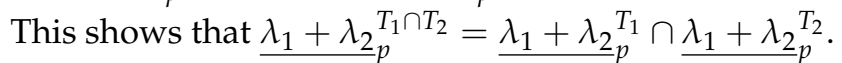

(4) This can be proved similar to (3). 
(5) Let $w_{1} \in W_{1}$. If $w_{1} \lambda_{1}(e) \cup w_{1} \lambda_{2}(e)=\varnothing$, then $p{\overline{\lambda_{1}+\lambda_{2}}}^{\mu_{T_{1}} \cup T_{2}}(e)\left(w_{1}\right)=0=p$ $\overline{\lambda_{1}+\lambda_{2}} \mu_{T_{1}}(e)\left(w_{1)} \cup^{p}{\overline{\lambda_{1}+\lambda_{2}}}^{\mu_{T_{2}}}(e)\left(w_{1}\right)\right.$

and $p \overline{\lambda_{1}+\lambda_{2}} \gamma_{T_{1} \cup T_{2}}(e)\left(w_{1}\right)=1=p{\overline{\lambda_{1}+\lambda_{2}}}^{\mu_{T_{1}}}(e)\left(w_{1)} \cap^{p}{\overline{\lambda_{1}+\lambda_{2}}}^{\mu_{T_{2}}}(e)\left(w_{1}\right)\right.$.

If $w_{1} \lambda_{1}(e) \cup w_{1} \lambda_{2}(e) \neq \varnothing$,

then $p \overline{\lambda_{1}+\lambda_{2}} \mu_{T_{1} \cup T_{2}}(e)\left(w_{1}\right)=\vee\left\{\left(\mu_{T_{1}} \vee \mu_{T_{2}}\right)\left(w_{2}\right): w_{2} \in\left(w_{1} \lambda_{1}(e) \cup w_{1} \lambda_{2}(e)\right)\right\}=$ $\vee\left\{\mu_{T_{1}}\left(w_{2}\right) \vee \mu_{T_{2}}\left(w_{2}\right): w_{2} \in\left(w_{1} \lambda_{1}(e) \cup w_{1} \lambda_{2}(e)\right)\right\}$

$=\left(\vee\left\{\mu_{T_{1}}\left(w_{2}\right): w_{2} \in\left(w_{1} \lambda_{1}(e) \cup w_{1} \lambda_{2}(e)\right)\right\}\right) \vee\left(\vee\left\{\mu_{T_{2}}\left(w_{2}\right): w_{2} \in\left(w_{1} \lambda_{1}(e) \cup\right.\right.\right.$ $\left.\left.\left.w_{1} \lambda_{2}(e)\right)\right\}\right)$

$=p{\overline{\lambda_{1}+\lambda_{2}}}^{\mu_{T_{1}}}(e)\left(w_{1}\right) \cup^{p}{\overline{\lambda_{1}+\lambda_{2}}}^{\mu_{T}}(e)\left(w_{1}\right)$.

In addition, $p \overline{\lambda_{1}+\lambda_{2}} \gamma_{T_{1} \cup T_{2}}(e)\left(w_{1}\right)=\wedge\left\{\left(\gamma_{T_{1}} \wedge \gamma_{T_{2}}\right)\left(w_{2}\right): w_{2} \in\left(w_{1} \lambda_{1}(e) \cap w_{1} \lambda_{2}(e)\right)\right\}$ $=\wedge\left\{\gamma_{T_{1}}\left(w_{2}\right) \wedge \gamma_{T_{2}}\left(w_{2}\right): w_{2} \in\left(w_{1} \lambda_{1}(e) \cap w_{1} \lambda_{2}(e)\right)\right\}=\left(\wedge\left\{\gamma_{T_{1}}\left(w_{2}\right): w_{2} \in\left(w_{1} \lambda_{1}(e) \cap\right.\right.\right.$ $\left.\left.\left.w_{1} \lambda_{2}(e)\right)\right\}\right) \wedge\left(\wedge\left\{\gamma_{T_{2}}\left(w_{2}\right): w_{2} \in\left(w_{1} \lambda_{1}(e) \cap w_{1} \lambda_{2}(e)\right)\right\}\right)$

$=p \bar{\lambda}_{1+\lambda_{2}} \gamma_{T_{1}}(e)\left(w_{1}\right) \cup^{p}{\overline{\lambda_{1}+\lambda_{2}}}^{\gamma_{T_{2}}}(e)\left(w_{1}\right)$.

This shows that $p \overline{\lambda_{1}+\lambda_{2}} T_{1} \cup T_{2}=p \frac{(e)\left(\lambda_{1}\right)}{\lambda_{1}+\lambda_{2}} \cup^{p} \overline{\lambda_{1}+\lambda_{2}} T_{2}$.

(6) This can be proved similar to (5).

For the converses of part (4) and (6), we have the following example.

Example 3. Let $W_{1}=\{a, b, c, d\}, W_{2}=\{1,2,3,4\}$ and $A=\left\{e_{1}, e_{2}\right\}$, and $\left(\lambda_{1}, A\right)$ and $\left(\lambda_{2}, A\right)$ be soft binary relations from $W_{1}$ to $W_{2}$ defined by

$$
\begin{aligned}
& \lambda_{1}\left(e_{1}\right)=\{(a, 1),(a, 3),(b, 2),(c, 3),(c, 4),(d, 1),(d, 4)\}, \\
& \lambda_{1}\left(e_{2}\right)=\{(a, 2),(a, 3),(b, 4),(c, 3),(d, 1),(d, 2),(d, 3)\}, \\
& \lambda_{2}\left(e_{1}\right)=\{(a, 4),(b, 3),(c, 1),(c, 2),(c, 3)\}, \\
& \lambda_{2}\left(e_{2}\right)=\{(b, 1),(b, 2),(b, 3),(c, 1),(c, 4),(d, 4)\}
\end{aligned}
$$

Then, their aftersets are

$$
\begin{array}{ll}
a \lambda_{1}\left(e_{1}\right)=\{1,3\}, & b \lambda_{1}\left(e_{1}\right)=\{2\}, \quad c \lambda_{1}\left(e_{1}\right)=\{3,4\}, \quad d \lambda_{1}\left(e_{1}\right)=\{1,4\}, \\
a \lambda_{1}\left(e_{2}\right)=\{2,3\}, \quad b \lambda_{1}\left(e_{2}\right)=\{4\}, \quad c \lambda_{1}\left(e_{2}\right)=\{3\}, \quad d \lambda_{1}\left(e_{2}\right)=\{1,2,3\}, \text { and } \\
a \lambda_{2}\left(e_{1}\right)=\{4\}, \quad b \lambda_{2}\left(e_{1}\right)=\{3\}, \quad c \lambda_{2}\left(e_{1}\right)=\{1,2,3\}, d \lambda_{2}\left(e_{1}\right)=\varnothing, \\
a \lambda_{2}\left(e_{2}\right)=\varnothing, \quad b \lambda_{2}\left(e_{2}\right)=\{1,2,3\}, c \lambda_{2}\left(e_{2}\right)=\{1,4\}, d \lambda_{2}\left(e_{2}\right)=\{4\} .
\end{array}
$$

(1) Define $T_{1}=\left\langle\mu_{T_{1}}, \gamma_{T_{1}}\right\rangle: W_{2} \rightarrow[0,1]$ as given in Table 5:

Table 5. Intuitionistic fuzzy set $T_{1}$.

\begin{tabular}{lllll}
\hline $\mathbf{T}_{\mathbf{1}}$ & $\mathbf{1}$ & $\mathbf{2}$ & $\mathbf{3}$ & $\mathbf{4}$ \\
\hline$\left(\mu_{T_{1}}, \gamma_{T_{1}}\right)$ & $(0.6,0.3)$ & $(0.4,0.5)$ & $(0.1,0.9)$ & $(0.3,0.6)$ \\
\hline
\end{tabular}

(2) Define $T_{2}=\left\langle\mu_{T_{2}}, \gamma_{T_{2}}\right\rangle: W_{2} \rightarrow[0,1]$ as given in Table 6:

Table 6. Intuitionistic fuzzy set $T_{2}$.

\begin{tabular}{lllll}
\hline $\boldsymbol{T}_{\mathbf{2}}$ & $\mathbf{1}$ & $\mathbf{2}$ & $\mathbf{3}$ & $\mathbf{4}$ \\
\hline$\left(\mu_{T_{2}}, \gamma_{T_{2}}\right)$ & $(0.3,0.7)$ & $(1.0,0.0)$ & $(0.4,0.5)$ & $(0.2,0.7)$ \\
\hline
\end{tabular}

(3) Define $T_{1} \cup T_{2}$ and $T_{1} \cap T_{2}$ as given in Tables 7 and 8 . 
Table 7. Union of $T_{1}$ and $T_{2}$.

\begin{tabular}{lllll}
\hline $\boldsymbol{T}_{\mathbf{1}} \cup \boldsymbol{T}_{\mathbf{2}}$ & $\mathbf{1}$ & $\mathbf{2}$ & $\mathbf{3}$ & $\mathbf{4}$ \\
\hline$\left(\mu_{T_{1} \cup T_{2}}, \gamma_{T_{1} \cup T_{2}}\right)$ & $(0.6,0.3)$ & $(1.0,0.0)$ & $(0.4,0.5)$ & $(0.3,0.6)$ \\
\hline
\end{tabular}

Table 8. Intersection of $T_{1}$ and $T_{2}$.

\begin{tabular}{lllll}
\hline $\boldsymbol{T}_{\mathbf{1}} \cap \boldsymbol{T}_{\mathbf{2}}$ & $\mathbf{1}$ & $\mathbf{2}$ & $\mathbf{3}$ & $\mathbf{4}$ \\
\hline$\left(\mu_{T_{1} \cap T_{2}}, \gamma_{T_{1} \cap T_{2}}\right)$ & $(0.3,0.7)$ & $(0.4,0.5)$ & $(0.1,0.9)$ & $(0.2,0.7)$ \\
\hline
\end{tabular}

The pessimistic multigranulation lower and upper approximations of $T_{1}, T_{2}, T_{1} \cup T_{2}$ and $T_{1} \cap T_{2}$ with respect to the aftersets are given in Table 9 .

Table 9. Pessimistic multigranulation lower and upper approximations of $T_{1}, T_{2}, T_{1} \cup T_{2}$ and $T_{1} \cap T_{2}$.

\begin{tabular}{|c|c|c|c|c|}
\hline & 1 & 2 & 3 & 4 \\
\left.\hline${\underline{\lambda_{1}+\lambda_{2}}}_{p}^{\mu_{T_{1}}}\left(e_{1}\right),{\underline{\lambda_{1}}+\lambda_{2}}_{p}^{\gamma_{T_{1}}}\left(e_{1}\right)\right)$ & $(1,0.9)$ & $(1,0.9)$ & $(0.1,0.9)$ & $(1.0,0.6)$ \\
\hline$\left({\underline{\lambda_{1}}+\lambda_{2}}_{p}^{\mu_{T_{1}}}\left(e_{2}\right),{\underline{\lambda_{1}}+\lambda_{2}}_{p}^{\gamma_{T_{1}}}\left(e_{2}\right)\right)$ & $(1.0,0.9)$ & $(1,0.9)$ & $(1.0,0.9)$ & $(1.0,0.9)$ \\
\hline 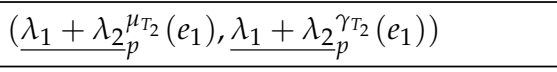 & $(1.0,0.7)$ & $(1.0,0.5)$ & $(0.4,0.7)$ & $(1.0,0.7)$ \\
\hline$\left({\underline{\lambda_{1}}+\lambda_{2}}_{p}^{\mu_{T_{2}}}\left(e_{2}\right), \underline{\lambda}_{1}+\lambda_{2}{ }_{p}^{\gamma_{T_{2}}}\left(e_{2}\right)\right)$ & $(1.0,0.5)$ & $(1.0,0.7)$ & $(1.0,0.7)$ & $(1.0,0.7)$ \\
\hline$\left(\underline{\lambda_{1}+\lambda_{2}}{ }_{p}^{\mu_{T_{1} \cup T_{2}}}\left(e_{1}\right), \underline{\lambda_{1}+\lambda_{2}}{ }_{p}^{\left.\gamma_{T_{1} \cup T_{2}}\left(e_{1}\right)\right)}\right.$ & $(1.0,0.3)$ & $(1.0,0.0)$ & $(0.4,0.0)$ & $(1.0,0.3)$ \\
\hline$\left({\underline{\lambda_{1}}+\lambda_{2}}_{p}^{\mu_{T_{1} \cup T_{2}}}\left(e_{2}\right), \underline{\lambda}_{1}+\lambda_{2}{ }_{p}^{\gamma T_{1} \cup T_{2}}\left(e_{2}\right)\right)$ & $(1.0,0.0)$ & $(1.0,0.0)$ & $(1.0,0.3)$ & $(1.0,0.0)$ \\
\hline$\left(p{\overline{\lambda_{1}+\lambda_{2}}}^{\mu_{T_{1}}}\left(e_{1}\right), p{\overline{\lambda_{1}+\lambda_{2}}}^{\gamma_{T_{1}}}\left(e_{1}\right)\right)$ & $(0.6,1.0)$ & $(0.4,1.0)$ & $(0.6,0.9)$ & $(0.6,1.0)$ \\
\hline$\left(p \overline{\lambda_{1}+\lambda_{2}}{ }^{\mu_{T_{1}}}\left(e_{2}\right), p{\overline{\lambda_{1}+\lambda_{2}}}^{\gamma_{T_{1}}}\left(e_{2}\right)\right)$ & $(0.4,1.0)$ & $(0.6,1)$ & $(0.6,1.0)$ & $(0.6,1.0)$ \\
\hline$\left(p{\overline{\lambda_{1}+\sigma_{2}}}^{\mu_{T_{2}}}\left(e_{1}\right),{ }^{p}{\overline{\lambda_{1}+\lambda_{2}}}^{\gamma_{T_{2}}}\left(e_{1}\right)\right)$ & $(0.4,1.0)$ & $(1.0,1.0)$ & $(1.0,0.5)$ & $(0.3,1.0)$ \\
\hline$\left(p{\overline{\lambda_{1}+\sigma_{2}}}^{\mu_{T_{2}}}\left(e_{2}\right), p{\overline{\lambda_{1}+\lambda_{2}}}_{T_{T_{2}}}\left(e_{2}\right)\right)$ & $(1.0,1.0)$ & $(1.0,1.0)$ & $(0.4,1.0)$ & $(1.0,1.0)$ \\
\hline$\left(p{\overline{\lambda_{1}+\sigma_{2}}}^{\mu_{T_{1} \cap T_{2}}}\left(e_{1}\right),{ }^{p}{\overline{\lambda_{1}+\lambda_{2}}}_{\gamma_{T_{1} \cap T_{2}}}\left(e_{1}\right)\right)$ & $(0.3,1.0)$ & $(0.4,1.0)$ & $(0.4,0.9)$ & $(0.3,1.0)$ \\
\hline 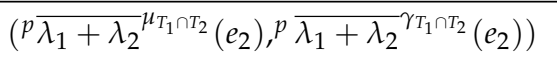 & $(0.4,1.0)$ & $(0.4,1.0)$ & $(0.3,1.0)$ & $(0.4,1.0)$ \\
\hline
\end{tabular}

From Table 9, we have the following

$$
\begin{aligned}
& {\underline{\lambda_{1}}+\lambda_{2}}_{p}^{\mu_{T_{1} \cup T_{2}}\left(e_{1}\right)(3)}=0.4=0.4={\underline{\lambda_{1}}+\lambda_{2}}_{p}^{\mu_{T_{1}}\left(e_{1}\right)(3) \vee}{\underline{\lambda_{1}}+\lambda_{2}}_{p}^{\mu_{T_{2}}}\left(e_{1}\right)(3) ; \text { but } \\
& \underline{\lambda_{1}+\lambda_{2}}{ }_{p}^{\gamma_{T_{1}} \cup T_{2}}\left(e_{1}\right)(3)=0.0 \ngtr 0.7=\underline{\lambda_{1}+\lambda_{2}^{2}}{ }_{p}^{\gamma_{T_{1}}}\left(e_{1}\right)(3) \wedge \underline{\lambda_{1}+\lambda_{2}}{ }_{p}^{\gamma_{T_{2}}}\left(e_{1}\right)(3) ;
\end{aligned}
$$

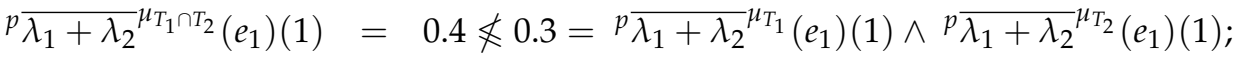

$$
\begin{aligned}
& p \overline{\lambda_{1}+\lambda_{2}} \gamma_{T_{1} \cap T_{2}}\left(e_{1}\right)(1)=1.0=1.0=p \overline{\lambda_{1}+\lambda_{2}} \gamma_{T_{1}}\left(e_{1}\right)(1) \vee p \overline{\lambda_{1}+\lambda_{2}} \gamma_{T_{2}}\left(e_{1}\right)(1) \text {. }
\end{aligned}
$$

Proposition 6. Let $\left(\lambda_{1}, A\right),\left(\lambda_{2}, A\right)$ be two soft relations from $W_{1}$ to $W_{2}$; that is, $\lambda_{1}: A \rightarrow$ $P\left(W_{1} \times W_{2}\right)$ and $\lambda_{2}: A \rightarrow P\left(W_{1} \times W_{2}\right)$ and $T, T_{1}, T_{2} \in I F\left(W_{1}\right)$. Then, the following properties hold.

(1) If $T_{1} \subseteq T_{2}$, then ${ }^{T_{1}} \underline{\lambda_{1}+\lambda_{2}} p \subseteq \subseteq^{T_{2}} \underline{\lambda_{1}+\lambda_{2}} p^{\prime}$;

(2) If $T_{1} \subseteq T_{2}$, then $T_{1}{\overline{\lambda_{1}+\lambda_{2}}}^{p} \subseteq T_{2}{\overline{\lambda_{1}+\lambda_{2}}}^{p}$;

(3) $T_{1} \cap T_{2} \underline{\lambda_{1}+\lambda_{2}} p={ }^{T_{1}} \underline{\lambda_{1}+\lambda_{2}} p \cap^{T_{2}} \underline{\lambda_{1}+\lambda_{2}} p^{\prime}$;

(4) $T_{1} \cup T_{2} \underline{\lambda_{1}+\lambda_{2}} p \supseteq^{T_{1}} \underline{\lambda_{1}+\lambda_{2}} p \cup^{T_{2}} \underline{\lambda_{1}+\lambda_{2}} p^{\prime}$;

(5) $T_{1} \cup T_{2}{\overline{\lambda_{1}+\lambda_{2}}}^{p}={ }^{T_{1}}{\overline{\bar{\lambda}_{1}+\lambda_{2}}}^{p} \cup^{T_{2}}{\overline{\lambda_{1}+\lambda_{2}}}^{p}$;

(6) $T_{1} \cap T_{2}{\overline{\lambda_{1}+\lambda_{2}}}^{p} \subseteq T_{1}{\overline{\lambda_{1}+\lambda_{2}}}^{p} \cap^{T_{2}}{\overline{\lambda_{1}+\lambda_{2}}}^{p}$.

Proof. The proof is similar to the proof of Proposition 5. 


\section{Roughness of an Intuitionistic Fuzzy Set over Two Universes by Multi Soft Relations}

In this Section, we discuss the pessimistic multigranulation roughness of an IFS by multi soft binary relations from $W_{1}$ to $W_{2}$ and approximate an IFS of universe $W_{2}$ in universe $W_{1}$ and an IFS $W_{1}$ in $W_{2}$ by using aftersets and foresets of soft binary relations, respectively. In this way, we obtain two intuitionistic fuzzy soft sets corresponding to IFSs in $W_{2}\left(W_{1}\right)$. We also study some properties of these approximations.

Definition 14. Let $W_{1}$ and $W_{2}$ be two non-empty finite universes and $\phi$ be a family of soft binary relations from $W_{1}$ to $W_{2}$. Then, we say $\left(W_{1}, W_{2}, \phi\right)$ is the multigranulation generalized soft approximation space over two universes.

Definition 15. Let $\left(W_{1}, W_{2}, \phi\right)$ be the multigranulation generalized soft approximation space over two universes $W_{1}$ and $W_{2}$, where $\phi=\lambda_{1}, \lambda_{2}, \lambda_{3}, \ldots . . \lambda_{n}$, and $T=\left\langle\mu_{T}, \gamma_{T}\right\rangle$ be an IFS in $W_{2}$. Then, the pessimistic multigranulation lower approximation $\underline{\sum_{i=1}^{n} \lambda_{i}^{T}}=\left(\underline{\sum_{i=1}^{n} \lambda_{i}}{ }_{p}^{\mu_{T}}, \sum_{i=1}^{n} \lambda_{i}^{\gamma_{T}}\right)$ and the pessimistic multigranulation upper approximation $p{\overline{\sum_{i=1}^{n} \lambda_{i}}}^{T}=\left(p{\overline{\sum_{i=1}^{n} \lambda_{i}}}^{\mu_{T}}, p{\overline{\sum_{i=1}^{n}}}_{\lambda_{i}}^{\gamma_{T}}\right)$ of $T=\left\langle\mu_{T}, \gamma_{T}\right\rangle$ are IF soft sets over $W_{1}$ with respect to the aftersets of soft relations $\left(\lambda_{i}, A\right) \in \phi$, and are defined as:

$$
\begin{aligned}
& {\underline{\sum_{i=1}^{n} \lambda_{i}^{\mu_{T}}}}_{p}(e)\left(w_{1}\right)=\left\{\begin{array}{cc}
\wedge\left\{\mu_{T}\left(w_{2}\right): w_{2} \in \cap_{i=1}^{n} w_{1} \lambda_{i}(e)\right\}, & \text { if } \cap_{i=1}^{n} w_{1} \lambda_{i}(e) \neq \varnothing ; \\
1 & \text { otherwise; }
\end{array}\right. \\
& \underline{\sum_{i=1}^{n} \lambda_{i}^{\gamma_{T}}}(e)\left(w_{1}\right)=\left\{\begin{array}{cc}
\vee\left\{\gamma_{T}\left(w_{2}\right): w_{2} \in \cup_{i=1}^{n} w_{1} \lambda_{i}(e)\right\}, & \text { if } \cup_{i=1}^{n} w_{1} \lambda_{i}(e) \neq \varnothing ; \\
0 & \text { otherwise; }
\end{array}\right.
\end{aligned}
$$

and

$$
\begin{aligned}
& p \overline{\sum_{i=1}^{n} \lambda_{i}} \mu_{T}(e)\left(w_{1}\right)=\left\{\begin{array}{cc}
\vee\left\{\mu_{T}\left(w_{2}\right): w_{2} \in \cup_{i=1}^{n} w_{1} \lambda_{i}(e)\right\}, & \text { if } \cup_{i=1}^{n} w_{1} \lambda_{i}(e) \neq \varnothing ; \\
0 & \text { otherwise; }
\end{array}\right. \\
& p \overline{\sum_{i=1}^{n} \lambda_{i}} \gamma_{T}(e)\left(w_{1}\right)=\left\{\begin{array}{cc}
\wedge\left\{\gamma_{T}\left(w_{2}\right): w_{2} \in \cap_{i=1}^{n} w_{1} \lambda_{i}(e)\right\}, & \text { if } \cap_{i=1}^{n} w_{1} \lambda_{i}(e) \neq \varnothing ; \\
1 & \text { otherwise. }
\end{array}\right.
\end{aligned}
$$

where $w_{1} \lambda_{i}(e)=\left\{w_{2} \in W_{2}:\left(w_{1}, w_{2}\right) \in \lambda_{i}(e)\right\}$ are called the aftersets of $w_{1}$ for $w_{1} \in W_{1}$ and $e \in A$. Obviously, $\left(\underline{\sum_{i=1}^{n} \lambda_{i}^{T}}{ }^{T}, A\right)$ and $\left({ }^{p}{\overline{\sum_{i=1}^{n}}}^{n}{ }_{i}{ }^{T}, A\right)$ are two IFS soft sets over $W_{1}$.

Definition 16. Let $\left(W_{1}, W_{2}, \phi\right)$ be the multigranulation generalized soft approximation space over two universes $W_{1}$ and $W_{2}$, where $\phi=\lambda_{1}, \lambda_{2}, \lambda_{3}, \ldots . . \lambda_{n}$, and $T=\left\langle\mu_{T}, \gamma_{T}\right\rangle$ be an IFS in $W_{1}$. Then, the pessimistic multigranulation lower approximation ${ }^{T} \underline{\sum_{i=1}^{n} \lambda_{i}} p=\left(\mu_{T}^{\sum_{i=1}^{n} \lambda_{i}}{ }^{\prime}{ }^{\gamma_{T}} \underline{\sum_{i=1}^{n} \lambda_{i}}\right)$ and the pessimistic multigranulation upper approximation ${ }^{T}{\overline{\sum_{i=1}^{n} \lambda_{i}}}^{p}=\left(\mu_{T}{\overline{\sum_{i=1}^{n} \lambda_{i}}}^{p}, \gamma_{T}{\overline{\sum_{i=1}^{n} \lambda_{i}}}^{p}\right)$ of $T=\left\langle\mu_{T}, \gamma_{T}\right\rangle$ are IF soft sets over $W_{2}$ with respect to the foresets of soft relations $\left(\lambda_{i}, A\right) \in \phi$, and are defined as:

$$
\begin{aligned}
& { }^{\mu_{T}{\underline{\sum_{i=1}^{n}}}_{\lambda_{i}}} p(e)\left(w_{2}\right)=\left\{\begin{array}{cc}
\wedge\left\{\mu_{T}\left(w_{1}\right): w_{1} \in \cap_{i=1}^{n} \lambda_{i}(e) w_{2}\right\}, & \text { if } \cap_{i=1}^{n} \lambda_{i}(e) w_{2} \neq \varnothing ; \\
1 & \text { otherwise; }
\end{array}\right. \\
& { }^{\gamma_{T}} \underline{\underline{\sum_{i=1}^{n} \lambda_{i}}} p(e)\left(w_{2}\right)=\left\{\begin{array}{cc}
\vee\left\{\gamma_{T}\left(w_{1}\right): w_{1} \in \cup_{i=1}^{n} \lambda_{i}(e) w_{2}\right\}, & \text { if } \begin{array}{c}
\cup_{i=1}^{n} \lambda_{i}(e) w_{2} \neq \varnothing ; \\
0
\end{array}
\end{array}\right.
\end{aligned}
$$

and

$$
\mu_{T}{\overline{\sum_{i=1}^{n} \lambda_{i}}}^{p}(e)\left(w_{2}\right)=\left\{\begin{array}{cc}
\vee\left\{\mu_{T}\left(w_{1}\right): w_{1} \in \cup_{i=1}^{n} \lambda_{i}(e) w_{2}\right\}, & \text { if } \cup_{i=1}^{n} \lambda_{i}(e) w_{2} \neq \varnothing ; \\
0 & \text { otherwise; }
\end{array}\right.
$$




$$
\gamma_{T}{\overline{\sum_{i=1}^{n} \lambda_{i}}}^{p}(e)\left(w_{2}\right)=\left\{\begin{array}{cc}
\wedge\left\{\gamma_{T}\left(w_{1}\right): w_{1} \in \cap_{i=1}^{n} \lambda_{i}(e) w_{2}\right\}, & \text { if } \cap_{i=1}^{n} \lambda_{i}(e) w_{2} \neq \varnothing ; \\
1 & \text { otherwise. }
\end{array}\right.
$$

where $\lambda_{1}(e) w_{2}=\left\{w_{1} \in W_{1}:\left(w_{1}, w_{2}\right) \in \lambda_{i}(e)\right\}$ are called the foresets of $w_{2}$ for $w_{2} \in W_{2}$ and $e \in A$. Obviously, $\left({ }^{T}{\underline{\sum_{i=1}^{n}} \lambda_{i}}_{p}{ }^{\prime} A\right)$ and $\left({ }^{T}{\overline{\sum_{i=1}^{n} \lambda_{i}}}^{p}, A\right)$ are two IFS soft sets over $W_{2}$.

Moreover, $\underline{\sum_{i=1}^{n} \lambda_{i}^{T}}: A \rightarrow \operatorname{IF}\left(W_{1}\right),{ }^{p}{\overline{\sum_{i=1}^{n} \lambda_{i}}}^{T}: A \rightarrow \operatorname{IF}\left(W_{1}\right)$ and ${ }^{T} \underline{\sum}_{i=1}^{n} \lambda_{i} p: A \rightarrow$ $\operatorname{IF}\left(W_{2}\right){ }^{T}{\overline{\sum_{i=1}^{n} \lambda_{i}}}^{p}: A \rightarrow \operatorname{IF}\left(W_{2}\right)$.

Proposition 7. Let $\left(W_{1}, W_{2}, \phi\right)$ be the multigranulation generalized soft approximation space over two universes $W_{1}$ and $W_{2}$ and $T=\left\langle\mu_{T}, \gamma_{T}\right\rangle$ be an IFS in $W_{2}$. Then, the following properties for $\sum_{i=1}^{n} \lambda_{i}^{T} p^{\prime} p{\overline{\sum_{i=1}^{n} \lambda_{i}}}^{T}$ hold.

(1) $\sum_{i=1}^{n} \lambda_{i}^{T} p \wedge_{i=1}^{n} \underline{\lambda}_{i}^{T}$;

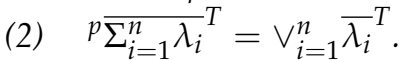

Proof. The proof is similar to the proof of Proposition 1.

Proposition 8. Let $\left(W_{1}, W_{2}, \phi\right)$ be the multigranulation generalized soft approximation space over two universes $W_{1}$ and $W_{2}$ and $T=\left\langle\mu_{T}, \gamma_{T}\right\rangle$ be an IFS in $W_{1}$. Then, the following properties for ${ }^{T} \sum_{i=1}^{n} \lambda_{i}{ }^{\prime}{ }^{T}{\overline{\sum_{i=1}^{n} \lambda_{i}}}^{p}$ hold.

(1) ${ }^{T} \underline{\sum}_{i=1}^{n} \lambda_{i} p \supseteq \wedge_{i=1}^{n}{ }^{T} \underline{\lambda_{i}}$;

(2) $T{\overline{\sum_{i=1}^{n} \lambda_{i}}}^{p}={ }_{i=1}^{n} \vee T \bar{\lambda}_{i}$.

Proof. The proof is similar to the proof of Proposition 2.

Proposition 9. Let $\left(W_{1}, W_{2}, \phi\right)$ be the multigranulation generalized soft approximation space over two universes $W_{1}$ and $W_{2}$. Then, the following properties with respect to the aftersets hold.

(1) ${\underline{\sum_{i=1}^{n}}}_{\lambda_{i}}^{{ }^{1} W_{2}}=1_{W_{1}}$ for all $e \in A$;

(2) $p \overline{\sum_{i=1}^{n} \lambda_{i}} 1_{W_{2}}=1_{W_{1}}$ if $\cup_{i=1}^{n} w_{1} \lambda_{i}(e) \neq \varnothing$, and $\cap_{i=1}^{n} w_{1} \lambda_{i}(e) \neq \varnothing$ for some $i \leq n$;

(3) $\sum_{i=1}^{n} \lambda_{i}{ }^{{ }_{W_{2}}}=0_{W_{1}}$ if $\cap_{i=1}^{n} w_{1} \lambda_{i}(e) \neq \varnothing$ and $\cup_{i=1}^{n} w_{1} \lambda_{i}(e) \neq \varnothing$, for some $i \leq n$; $p \bar{\sum}_{i=1}^{n} \lambda_{i}{ }^{W_{2}}=0_{W_{1}}$ for all $e \in A$.

Proof. The proof is similar to the proof of Proposition 3.

Proposition 10. Let $\left(W_{1}, W_{2}, \phi\right)$ be the multigranulation generalized soft approximation space over two universes $W_{1}$ and $W_{2}$. Then, the following properties with respect to the foresets hold.

(1) $1_{W_{1}} \underline{\sum_{i=1}^{n} \lambda_{i}} p=1_{W_{2}}$ for all $e \in A$;

(2) $1_{W_{1}}{\overline{\sum_{i=1}^{n} \lambda_{i}}}^{p}=1_{W_{2}}$ if $\cup_{i=1}^{n} \lambda_{i}(e) w_{2} \neq \varnothing$ and $\cap_{i=1}^{n} \lambda_{i}(e) w_{2} \neq \varnothing$ for some $i \leq n$;

(3) $\quad 0_{W_{1}} \sum_{i=1}^{n} \lambda_{i}=0_{W_{2}}$ if $\cap_{i=1}^{n} \lambda_{i}(e) w_{2} \neq \varnothing$ and $\cup_{i=1}^{n} \lambda_{i}(e) w_{2} \neq \varnothing$, for some $i \leq n$;

(4) $0_{W_{1}}{\overline{\bar{\Sigma}_{i=1}^{n} \lambda_{i}}}^{p}=0_{W_{2}}$ for all $e \in A$.

Proof. The proof is similar to the proof of Proposition 4.

Proposition 11. Let $\left(W_{1}, W_{2}, \phi\right)$ be the multigranulation generalized soft approximation space over two universes $W_{1}$ and $W_{2}$ and $T, T_{1}, T_{2} \in I F\left(W_{2}\right)$. Then, the following properties for $\underline{\sum_{i=1}^{n} \lambda_{i}{ }^{T}}{ }^{\prime}{ }^{p}{\overline{\sum_{i=1}^{n} \lambda_{i}}}^{T}$ with respect to the aftersets hold. 
(1) If $T_{1} \subseteq T_{2}$, then $\underline{\sum_{i=1}^{n} \lambda_{i}^{T_{1}}} \subseteq \underline{\sum_{i=1}^{n} \lambda_{i}^{T_{2}}} p^{T_{2}}$;

(2) If $T_{1} \subseteq T_{2}$, then $p{\overline{\sum_{i=1}^{n} \lambda_{i}}}^{T} \subseteq p{\overline{\sum_{i=1}^{n}} \lambda_{i}^{T}}^{T_{2}}$;

(3) $\quad \underline{\sum_{i=1}^{n} \lambda_{i}^{T_{1}} p T_{2}}=\underline{\sum_{i=1}^{n} \lambda_{i}^{T_{1}}} p \underline{\sum_{i=1}^{n} \lambda_{i}^{T_{2}}} p$;

(4) $\underline{\sum_{i=1}^{n} \lambda_{i}^{T_{1}}}{ }_{p}^{T_{2}} \supseteq T_{2} \supseteq \underline{\sum_{i=1}^{n} \lambda_{i}^{T_{1}}} \cup \underline{\sum_{i=1}^{n} \lambda_{i}^{T_{2}}} p^{T_{2}}$;

(5) $p \overline{\sum_{i=1}^{n} \lambda_{i}} T_{1} \cup T_{2}={ }^{p}{\overline{\sum_{i=1}^{n} \lambda_{i}}}^{T_{1}} \cup^{p}{\overline{\sum_{i=1}^{n}} \lambda_{i}}^{T_{2}}$;

(6) $p \sum_{i=1}^{n} \lambda_{i} T_{1} \cap T_{2} \subseteq^{p} \sum_{i=1}^{n} \lambda_{i}{ }^{T} \cap^{p} \sum_{i=1}^{n} \lambda_{i}{ }^{T}$.

Proof. The proof is similar to the proof of Proposition 5.

Proposition 12. Let $\left(W_{1}, W_{2}, \phi\right)$ be the multigranulation generalized soft approximation space over two universes $W_{1}$ and $W_{2}$ and $T, T_{1}, T_{2} \in \operatorname{IF}\left(W_{1}\right)$. Then, the following properties for ${ }^{T}{\underline{\sum_{i=1}^{n} \lambda_{i}}}_{p^{\prime}}{ }^{T}{\overline{\sum_{i=1}^{n} \lambda_{i}}}^{p}$ with respect to the foresets hold.

(1) If $T_{1} \subseteq T_{2}$, then $T_{1} \underline{\sum_{i=1}^{n} \lambda_{i}} p \subseteq T^{T_{2}} \underline{\sum_{i=1}^{n} \lambda_{i}} p^{\text {; }}$

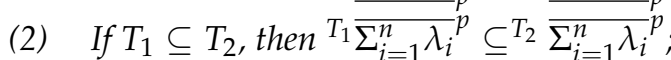

(3) $T_{1} \cap T_{2} \underline{\sum_{i=1}^{n} \lambda_{i}} p=T_{1} \underline{\sum_{i=1}^{n} \lambda_{i}} p \cap^{T_{2}} \underline{\sum_{i=1}^{n} \lambda_{i}} p^{\prime}$

(4) $T_{1} \cup T_{2} \underline{\sum_{i=1}^{n} \lambda_{i}} p \supseteq^{T_{1}} \underline{\sum_{i=1}^{n} \lambda_{i}} \cup^{T_{2}} \underline{\sum_{i=1}^{n} \lambda_{i}} p^{\prime}$

(5) ${ }^{T_{1} \cup T_{2}}{\overline{\sum_{i=1}^{n} \lambda_{i}}}^{p}={ }^{T_{1}}{\overline{\sum_{i=1}^{n} \lambda_{i}}}^{p} \cup^{T_{2}}{\overline{\sum_{i=1}^{n} \lambda_{i}}}^{p}$;

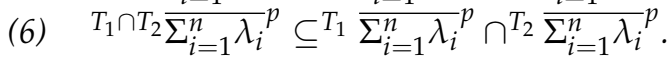

Proof. The proof is similar to the proof of Proposition 6.

Proposition 13. Let $\left(W_{1}, W_{2}, \phi\right)$ be the multigranulation generalized soft approximation space over two universes $W_{1}$ and $W_{2}$ and $T_{1}, T_{2}, T_{3}, \ldots . T_{m} \in I F\left(W_{2}\right)$, and $T_{1} \subseteq T_{2} \subseteq T_{3} \subseteq \ldots \subseteq T_{m}$. Then, the following properties with respect to the aftersets hold.

(1) $\underline{\sum_{i=1}^{n} \lambda_{i}^{T_{1}}} \subseteq \underline{\sum_{i=1}^{n} \lambda_{i}^{T_{2}}} \subseteq \underline{\sum_{i=1}^{n} \lambda_{i}^{T_{3}}} \underline{p} \subseteq \ldots . . \underline{\sum_{i=1}^{n} \lambda_{i}^{T_{m}}} p$;

(2) $p{\overline{\sum_{i=1}^{n} \lambda_{i}}}^{T_{1}} \subseteq^{p}{\overline{\sum_{i=1}^{n} \lambda_{i}}}^{T_{2}} \subseteq^{p}{\overline{\sum_{i=1}^{n}}}^{p} \lambda_{i}{ }^{T_{3}} \subseteq \ldots . \subseteq^{p}{\overline{\sum_{i=1}^{n}}}_{i}^{\lambda_{i}}{ }^{T}$.

Proof. The proof is similar to the proof of Proposition 5.

Proposition 14. Let $\left(W_{1}, W_{2}, \phi\right)$ be the multigranulation generalized soft approximation space over two universes $W_{1}$ and $W_{2}$ and $T_{1}, T_{2}, T_{3}, \ldots . T_{m} \in I F\left(W_{1}\right)$, and $T_{1} \subseteq T_{2} \subseteq T_{3} \subseteq \ldots \subseteq T_{m}$. Then, the following properties with respect to the foresets hold.

(1) $\quad T_{1} \underline{\sum_{i=1}^{n} \lambda_{i}} p \subseteq^{T_{2}} \underline{\sum_{i=1}^{n} \lambda_{i}} p \subseteq^{T_{3}} \underline{\sum_{i=1}^{n} \lambda_{i}} p \subseteq \ldots . . \subseteq^{T_{m}} \underline{\sum_{i=1}^{n} \lambda_{i}} p^{\prime}$

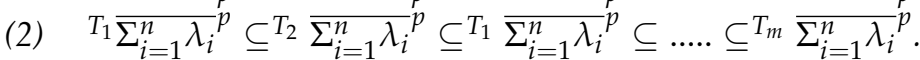

Proof. The proof is similar to the proof of Proposition 6.

\section{Application in Decision-Making Problem}

Data analysis is always needed to make a perception about any decision. Decision making is a good technique to study data analysis. Decision making is the process used to choose better alternatives from aspirants. A single expert's opinion is no longer preferable because a collective decision based on multiple expert' opinions is more effective. Shabir et al. [40] presented a decision-making algorithm using crisp sets. Jamal and Shabir [50] proposed a useful decision-making algorithm with the help of an OMGFRS model in terms of soft relations. The OMGIFRS model [41] is the extension of Jamal's OMGFRS model and corresponds to the decision-making method in terms of multi soft relations. In this paper, we present a suitable decision-making algorithm based on our proposed PMGIFRS model. 
The lower and upper approximations are the two subsets of a universe most close to being approximated. We obtain two corresponding values $\sum_{i=1}^{n} \lambda_{i}^{T}\left(e_{j}\right)\left(x_{k}\right)$ and $p \bar{\sum}_{i=1}^{n} \lambda_{i}^{T}\left(e_{j}\right)\left(x_{k}\right)$ with respect to the afterset of the decision alternative $x_{k} \in W_{1}$ by the IF soft lower and upper approximations of an IF $T \in \operatorname{IF}\left(W_{2}\right)$.

We present two algorithms for our proposed model here.

Algorithm 1 is presented with respect to the aftersets for the decision-making problem in the following.

\section{Algorithm 1 Respect to the aftersets for the decision-making problem}

(1) Compute the pessimistic multigranulation lower IF soft set approximation $\underline{\sum_{i=1}^{n} \lambda_{i}^{T}} p$ and pessimistic multigranulation upper IF soft set approximation $p{\overline{\sum_{i=1}^{n} \lambda_{i}}}^{T}$ of an IF set $T=\left\langle\mu_{T}, \gamma_{T}\right\rangle$ with respect to the aftersets.

(2) Compute the score values for each of the entries of the ${\underline{\sum_{i=1}^{n}}}_{\lambda_{i}}^{T}$ and ${ }^{p}{\overline{\sum_{i=1}^{n}}}_{\lambda_{i}}^{T}$ and denote them by $\underline{S}_{i j}\left(x_{i}, e_{j}\right)$ and $\bar{S}_{i j}\left(x_{i}, e_{j}\right)$ for all $i, j$.

(3) Compute the aggregated score $\underline{S}\left(x_{i}\right)=\sum_{j=1}^{m} \underline{S}_{i j}\left(x_{i}, e_{j}\right)$ and $\bar{S}\left(x_{i}\right)=\sum_{j=1}^{m} \bar{S}_{i j}\left(x_{i}, e_{j}\right)$.

(4) Compute $S\left(x_{i}\right)=\underline{S}\left(x_{i}\right)+\bar{S}\left(x_{i}\right)$.

(5) The best decision is $x_{k}=\max _{i} S\left(x_{i}\right)$.

(6) If $k$ has more than one value, say, $k_{1}, k_{2}$, then we calculate the accuracy values $\underline{H}_{i j}\left(x_{i}, e_{j}\right)$ and $\bar{H}_{i j}\left(x_{i}, e_{j}\right)$ for only those $x_{k}$ for which $S\left(x_{k}\right)$ are equal.

(7) Compute $H\left(x_{k}\right)=\sum_{j=1}^{m} \underline{H}_{k j}\left(x_{k}, e_{j}\right)+\sum_{j=1}^{m} \bar{H}_{k j}\left(x_{k}, e_{j}\right)$ for $k=k_{1}, k_{2}$.

(8) If $H\left(x_{k_{1}}\right)>H\left(x_{k_{2}}\right)$, then we select $x_{k_{1}}$.

(9) If $H\left(x_{k_{1}}\right)=H\left(x_{k_{2}}\right)$, then select any one of $x_{k_{1}}$ and $x_{k_{2}}$.

Algorithm 2 is presented with respect to the foresets for the decision-making problem in the following.

Now, the following example shows the decision-making approach step by step. This example discusses the decision-making algorithm to make wiser decisions for a coach.

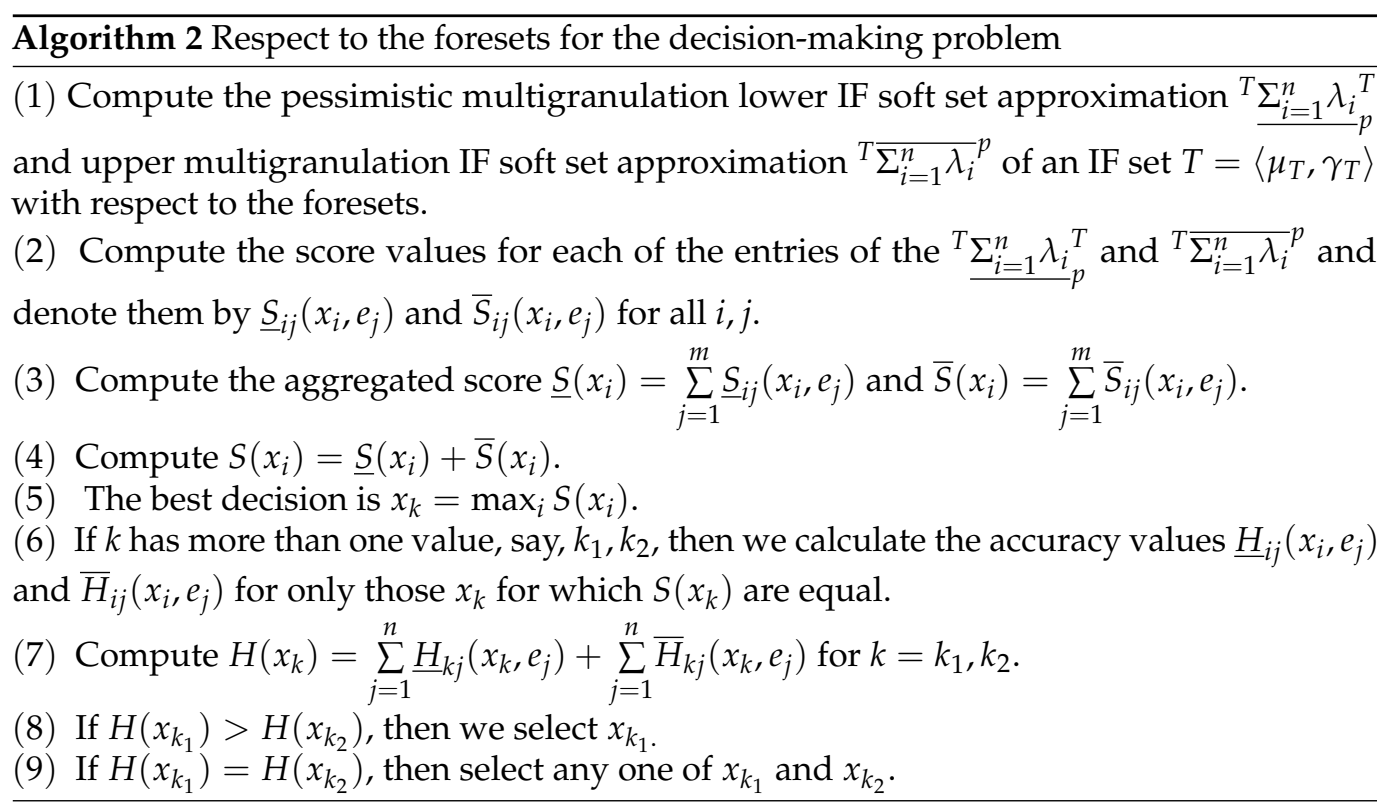


Example 4. Suppose that the Pakistan Cricket Board (PCB) wants to select a head coach and that there are 10 short-listed applicants, who are categorized in two groups: domestic and foreign. The set $W_{1}=\left\{d_{1}, d_{2}, d_{3}, d_{4}, d_{5}, d_{6}\right\}$ represents the applicants of the domestic group and $W_{2}=$ $\left\{f_{1}, f_{2}, f_{3}, f_{4}\right\}$ represents the applicants of the foreign group. Let $A=\left\{e_{1}, e_{2}, e_{3}\right\}$, where $\left\{e_{1}=\right.$ batting experience, $e_{2}=$ bowling experience, $e_{3}=$ managerial expertise $\}$ be the set of parameters. Let two different teams of interviewers analyze and compare these applicants for their competence.

We have $\lambda_{1}: A \rightarrow P\left(W_{1} \times W_{2}\right)$, which represents a comparison of the first-interviewer team defined by

$$
\begin{aligned}
\lambda_{1}\left(e_{1}\right) & =\left\{\left(d_{1}, f_{1}\right),\left(d_{1}, f_{2}\right),\left(d_{2}, f_{2}\right),\left(d_{2}, f_{3}\right),\left(d_{4}, f_{2}\right),\left(d_{4}, f_{3}\right),\left(d_{5}, f_{3}\right),\left(d_{6}, f_{1}\right)\right\} ; \\
\lambda_{1}\left(e_{2}\right) & =\left\{\left(d_{1}, f_{1}\right),\left(d_{2}, f_{3}\right),\left(d_{4}, f_{1}\right),\left(d_{5}, f_{1}\right),\left(d_{6}, f_{2}\right),\left(d_{6}, f_{3}\right)\right\} ; \\
\text { and } \lambda_{1}\left(e_{3}\right) & =\left\{\left(d_{1}, f_{1}\right),\left(d_{2}, f_{3}\right),\left(d_{3}, f_{1}\right),\left(d_{3}, f_{3}\right),\left(d_{4}, f_{1}\right),\left(d_{5}, f_{3}\right),\left(d_{6}, f_{1}\right)\right\} .
\end{aligned}
$$

where $\lambda_{1}\left(e_{1}\right)$ compares the batting experience of applicants, $\lambda_{1}\left(e_{2}\right)$ compares the bowling experience of applicants and $\lambda_{1}\left(e_{3}\right)$ compares the managerial expertise of applicants.

Similarly, $\lambda_{2}: A \rightarrow P\left(W_{1} \times W_{2}\right)$ represents a comparison of the second-interviewer team defined by

$$
\begin{aligned}
\lambda_{2}\left(e_{1}\right) & =\left\{\left(d_{1}, f_{1}\right),\left(d_{1}, f_{2}\right),\left(d_{2}, f_{3}\right),\left(d_{3}, f_{3}\right),\left(d_{4}, f_{2}\right),\left(d_{5}, f_{2}\right),\left(d_{6}, f_{3}\right)\right\} ; \\
\lambda_{2}\left(e_{2}\right) & =\left\{\left(d_{1}, f_{1}\right),\left(d_{3}, f_{2}\right),\left(d_{4}, f_{1}\right),\left(d_{6}, f_{2}\right)\right\} ; \\
\text { and } \lambda_{2}\left(e_{3}\right) & =\left\{\left(d_{1}, f_{1}\right),\left(d_{1}, f_{3}\right),\left(d_{2}, f_{2}\right),\left(d_{2}, f_{3}\right),\left(d_{4}, f_{1}\right),\left(d_{5}, f_{3}\right),\left(d_{6}, f_{1}\right)\right\} .
\end{aligned}
$$

where $\lambda_{2}\left(e_{1}\right)$ compares the batting experience of applicants, $\lambda_{2}\left(e_{2}\right)$ compares the bowling experience of applicants and $\lambda_{2}\left(e_{3}\right)$ compares the managerial expertise of applicants.

From these comparisons, we obtain two soft relations from $W_{1}$ to $W_{2}$. Now, the aftersets

$$
\begin{aligned}
& d_{1} \lambda_{1}\left(e_{1}\right)=\left\{f_{1}, f_{2}\right\}, d_{2} \lambda_{1}\left(e_{1}\right)=\left\{f_{2}, f_{3}\right\}, d_{3} \lambda_{1}\left(e_{1}\right)=\varnothing, \\
& d_{4} \lambda_{1}\left(e_{1}\right)=\left\{f_{2}, f_{3}\right\}, d_{5} \lambda_{1}\left(e_{1}\right)=\left\{f_{3}\right\}, \quad d_{6} \lambda_{1}\left(e_{1}\right)=\left\{f_{1}\right\} \text { and } \\
& d_{1} \lambda_{1}\left(e_{2}\right)=\left\{f_{1}\right\}, \quad d_{2} \lambda_{1}\left(e_{2}\right)=\left\{f_{3}\right\}, \quad d_{3} \lambda_{1}\left(e_{2}\right)=\varnothing, \\
& d_{4} \lambda_{1}\left(e_{2}\right)=\left\{f_{1}\right\}, \quad d_{5} \lambda_{1}\left(e_{2}\right)=\left\{f_{1}\right\}, \quad d_{6} \lambda_{1}\left(e_{2}\right)=\left\{f_{2}, f_{3}\right\}, \text { and } \\
& d_{1} \lambda_{1}\left(e_{3}\right)=\left\{f_{1}\right\}, \quad d_{2} \lambda_{1}\left(e_{3}\right)=\left\{f_{3}\right\}, \quad d_{3} \lambda_{1}\left(e_{3}\right)=\left\{f_{1}, f_{3}\right\}, \\
& d_{4} \lambda_{1}\left(e_{3}\right)=\left\{f_{1}\right\}, \quad d_{5} \lambda_{1}\left(e_{3}\right)=\left\{f_{3}\right\}, \quad d_{6} \lambda_{1}\left(e_{3}\right)=\left\{f_{1}\right\}, \text { and } \\
& d_{1} \lambda_{2}\left(e_{1}\right)=\left\{f_{1}, f_{2}\right\}, d_{2} \lambda_{2}\left(e_{1}\right)=\left\{f_{3}\right\}, \quad d_{3} \lambda_{2}\left(e_{1}\right)=\left\{f_{3}\right\}, \\
& d_{4} \lambda_{2}\left(e_{1}\right)=\left\{f_{2}\right\}, \quad d_{5} \lambda_{2}\left(e_{1}\right)=\left\{f_{2}\right\}, \quad d_{6} \lambda_{2}\left(e_{1}\right)=\left\{f_{3}\right\} \text { and } \\
& d_{1} \lambda_{2}\left(e_{2}\right)=\left\{f_{1}\right\}, \quad d_{2} \lambda_{2}\left(e_{2}\right)=\varnothing, \quad d_{3} \lambda_{2}\left(e_{2}\right)=\left\{f_{2}\right\}, \\
& d_{4} \lambda_{2}\left(e_{2}\right)=\left\{f_{1}\right\}, \quad d_{5} \lambda_{2}\left(e_{2}\right)=\varnothing, \quad d_{6} \lambda_{2}\left(e_{2}\right)=\left\{f_{2}\right\}, \text { and } \\
& d_{1} \lambda_{2}\left(e_{3}\right)=\left\{f_{1}, f_{3}\right\}, d_{2} \lambda_{2}\left(e_{3}\right)=\left\{f_{2}, f_{3}\right\}, d_{3} \lambda_{2}\left(e_{3}\right)=\varnothing, \\
& d_{4} \lambda_{2}\left(e_{3}\right)=\left\{f_{1}\right\}, \quad d_{5} \lambda_{2}\left(e_{3}\right)=\left\{f_{3}\right\}, \quad d_{6} \lambda_{2}\left(e_{3}\right)=\left\{f_{1}\right\},
\end{aligned}
$$

where $d_{i} \lambda_{j}\left(e_{1}\right)$ represents all those applicants of the domestic group whose batting experience is equal to $d_{i}, d_{i} \lambda_{j}\left(e_{2}\right)$ represents all those applicants of the domestic group whose bowling experience is equal to $d_{i}$ and $d_{i} \lambda_{j}\left(e_{3}\right)$ represents all those applicants of the domestic group whose managerial expertise is equal to $m_{i}$. In addition, foresets

$$
\begin{aligned}
& \lambda_{1}\left(e_{1}\right) f_{1}=\left\{d_{1}, d_{6}\right\}, \quad \lambda_{1}\left(e_{1}\right) f_{2}=\left\{d_{1}, d_{2}, d_{4}\right\}, \lambda_{1}\left(e_{1}\right) f_{3}=\left\{d_{2}, d_{4}, d_{5}\right\}, \text { and } \\
& \lambda_{1}\left(e_{2}\right) f_{1}=\left\{d_{1}, d_{4}, d_{5}\right\}, \quad \lambda_{1}\left(e_{2}\right) f_{2}=\left\{d_{6}\right\}, \quad \lambda_{1}\left(e_{2}\right) f_{3}=\left\{d_{2}, d_{6}\right\}, \text { and } \\
& \lambda_{1}\left(e_{3}\right) f_{1}=\left\{d_{1}, d_{3}, d_{4}, d_{6}\right\}, \lambda_{1}\left(e_{3}\right) f_{2}=\varnothing, \quad \lambda_{1}\left(e_{3}\right) f_{3}=\left\{d_{2}, d_{3}, d_{5}\right\} . \\
& \lambda_{2}\left(e_{1}\right) f_{1}=\left\{d_{1}\right\}, \quad \lambda_{2}\left(e_{1}\right) f_{2}=\left\{d_{1}, d_{4}, d_{5}\right\}, \lambda_{2}\left(e_{1}\right) f_{3}=\left\{d_{2}, d_{3}, d_{6}\right\} \text {, and } \\
& \lambda_{2}\left(e_{2}\right) f_{1}=\left\{d_{1}, d_{4}\right\}, \quad \lambda_{2}\left(e_{2}\right) f_{2}=\left\{d_{3}, d_{6}\right\}, \quad \lambda_{2}\left(e_{2}\right) f_{3}=\varnothing \text {, and } \\
& \lambda_{2}\left(e_{3}\right) f_{1}=\left\{d_{1}, d_{4}, d_{6}\right\}, \quad \lambda_{2}\left(e_{3}\right) f_{2}=\left\{d_{2}\right\}, \quad \lambda_{1}\left(e_{3}\right) f_{3}=\left\{d_{1}, d_{2}, d_{5}\right\} .
\end{aligned}
$$


where $\lambda_{j}\left(e_{1}\right) f_{i}$ represents all those applicants of the foreign group whose batting experience is equal to $f_{i}, \lambda_{j}\left(e_{2}\right) f_{i}$ represents all those applicants of the foreign group whose bowling experience is equal to $f_{i}$ and $\lambda_{j}\left(e_{3}\right) f_{i}$ represents all those applicants of the foreign group whose managerial expertise is equal to $f_{i}$.

Define $T=\left\langle\mu_{T}, \gamma_{T}\right\rangle: W_{2} \rightarrow[0,1]$, which represents the preference of applicants given by PCB such that $\mu_{T}\left(f_{1}\right)=0.2, \mu_{T}\left(f_{2}\right)=0.1, \mu_{T}\left(f_{3}\right)=0.5$, and $\gamma_{T}\left(f_{1}\right)=0.7, \gamma_{T}\left(f_{2}\right)=0.9, \gamma_{T}\left(f_{3}\right)=0.4$.

Define $T_{1}=\left\langle\mu_{T_{1}}, \gamma_{T_{1}}\right\rangle: W_{1} \rightarrow[0,1]$, which represents the preference of applicants given by PCB such that $\mu_{T_{1}}\left(d_{1}\right)=0.9, \mu_{T_{1}}\left(d_{2}\right)=0.6, \mu_{T_{1}}\left(d_{3}\right)=0.8, \mu_{T_{1}}\left(d_{4}\right)=0.3$,

$\mu_{T_{1}}\left(d_{5}\right)=0.9, \mu_{T_{1}}\left(d_{6}\right)=0.7$ and

$\gamma_{T_{1}}\left(d_{1}\right)=0, \gamma_{T_{1}}\left(d_{2}\right)=0.3, \gamma_{T_{1}}\left(d_{3}\right)=0.1, \gamma_{T_{1}}\left(d_{4}\right)=0.6$

$\gamma_{T_{1}}\left(d_{5}\right)=0.1, \gamma_{T_{1}}\left(d_{6}\right)=0.2$.

Therefore, the pessimistic multigranulation lower and upper approximations (with respect to the aftersets, as well as with respect to the foresets) are

$$
\begin{aligned}
\frac{\lambda_{1}+\lambda_{2}^{T}}{p} & =\left({\underline{\lambda_{1}+\lambda_{2}}}_{p}^{\mu_{T}}, \lambda_{1}+\lambda_{2}{ }_{0}^{\gamma_{T}}\right) \text { (given in Table 10) } \\
p{\overline{\lambda_{1}+\lambda_{2}}}^{T} & =\left({ }^{p}{\overline{\lambda_{1}+\lambda_{2}}}^{\mu_{T}}, p{\overline{\lambda_{1}+\lambda_{2}}}^{\gamma}\right) \text { (given in Table 11), where }
\end{aligned}
$$

\begin{tabular}{|c|c|c|c|c|c|c|}
\hline$\underline{\lambda_{1}+\lambda_{2}}{ }_{p}^{\mu_{T}},{\underline{\lambda_{1}}+\lambda_{2}^{\gamma_{T}}}_{p}^{\gamma_{T}}$ & $d_{1}$ & $d_{2}$ & $d_{3}$ & $d_{4}$ & $d_{5}$ & $d_{6}$ \\
\hline$\underline{\lambda_{1}+\lambda_{2}}{ }_{p}^{\mu_{T}}\left(e_{1}\right), \underline{\lambda}_{1}+\lambda_{2}^{\gamma_{T}}\left(e_{1}\right)$ & $(0.1,0.9)$ & $(1,0.4)$ & $(0.1,0.9)$ & $(0.1,0.9)$ & $(1,0.9)$ & $(1,0.7)$ \\
\hline$\lambda_{1}+\lambda_{2}^{2}{ }_{p}^{\mu_{T}}\left(e_{2}\right), \underline{\lambda}_{1}+\lambda_{2}^{\gamma_{p}}{ }_{p}^{\gamma_{T}}\left(e_{2}\right)$ & $(0.2,0.7)$ & $(1,0.4)$ & $(1,0.9)$ & $(0.2,0.7)$ & $(1,0.4)$ & $(0.1,0.4)$ \\
\hline$\lambda_{1}+\lambda_{2}^{2}{ }_{p}^{\mu_{T}}\left(e_{3}\right), \lambda_{1}+\lambda_{2}^{\gamma_{T}}{ }_{p}^{\gamma_{T}}\left(e_{3}\right)$ & $(0.2,0.7)$ & $(0.5,0.9)$ & $(1,0.7)$ & $(0.2,0.7)$ & $(0.5,0.4)$ & $(0.2,0.7)$ \\
\hline
\end{tabular}

Table 10. Pessimistic multigranulation lower approximations of $T$.

Table 10 shows the exact degree of the competency of the applicant $d_{i}$ to $T$ in their batting experience, bowling experience and managerial expertise.

Table 11. Pessimistic multigranulation upper approximations of $T$.

\begin{tabular}{lllllll}
\hline$p{\overline{\lambda_{\mathbf{1}}+\lambda_{\mathbf{2}}}}^{\mu_{T}},{ }^{p}{\overline{\lambda_{\mathbf{1}}+\lambda_{\mathbf{2}}}}^{\gamma_{T}}$ & $\boldsymbol{d}_{\mathbf{1}}$ & $\boldsymbol{d}_{\mathbf{2}}$ & $\boldsymbol{d}_{\mathbf{3}}$ & $\boldsymbol{d}_{\mathbf{4}}$ & $\boldsymbol{d}_{\mathbf{5}}$ & $\boldsymbol{d}_{\boldsymbol{6}}$ \\
\hline$p{\overline{\lambda_{1}+\lambda_{2}}}^{\mu_{T}}\left(e_{1}\right),{ }^{p}{\overline{\lambda_{1}+\lambda_{2}}}^{\gamma_{T}}\left(e_{1}\right)$ & $(0.2,0.7)$ & $(0.5,0.4)$ & $(0.5,1)$ & $(0.5,0.9)$ & $(0.5,1)$ & $(0.5,1)$ \\
\hline$p{\overline{\lambda_{1}+\lambda_{2}}}^{\mu_{T}}\left(e_{2}\right),{ }^{p}{\overline{\lambda_{1}+\lambda_{2}}}^{\gamma_{T}}\left(e_{2}\right)$ & $(0.2,0.7)$ & $(0.5,1)$ & $(0.1,1)$ & $(0.2,0.7)$ & $(0.2,1)$ & $(0.5,0.9)$ \\
\hline$p{\overline{\lambda_{1}+\lambda_{2}}}^{\mu_{T}}\left(e_{3}\right),{ }^{p}{\overline{\lambda_{1}+\lambda_{2}}}^{\gamma_{T}}\left(e_{3}\right)$ & $(0.5,0.7)$ & $(0.5,0.4)$ & $(0.5,1)$ & $(0.2,0.7)$ & $(0.5,0.4)$ & $(0.2,0.7)$ \\
\hline
\end{tabular}

Table 11 shows the possible degree of competency of applicant $d_{i}$ to $T$ in their batting experience, bowling experience and managerial expertise.

It is shown in Table 12 that $S\left(d_{5}\right)=3.1$ is the maximum, so PCB will select applicant $d_{5}$. 
Table 12. Values of score function of applicants.

\begin{tabular}{llllllllll}
\hline & $\underline{S}_{i j}\left(e_{1}\right)$ & $\underline{S}_{i j}\left(e_{2}\right)$ & $\underline{S}_{i j}\left(e_{3}\right)$ & $\bar{S}_{i j}\left(e_{1}\right)$ & $\bar{S}_{i j}\left(e_{2}\right)$ & $\bar{S}_{i j}\left(e_{3}\right)$ & $\underline{S}\left(x_{i}\right)$ & $\bar{S}\left(x_{i}\right)$ & $S\left(x_{i}\right)$ \\
\hline$d_{1}$ & -0.8 & -0.5 & -0.5 & -0.5 & -0.5 & -0.2 & -1.8 & -1.2 & -3.0 \\
\hline$d_{2}$ & 0.6 & 0.6 & -0.4 & 0.1 & -0.5 & 0.1 & 0.8 & -0.3 & 0.5 \\
\hline$d_{3}$ & -0.8 & 0.1 & 0.3 & 0.4 & -0.9 & 0.4 & -0.4 & -0.1 & -0.5 \\
\hline$d_{4}$ & -0.8 & -0.5 & -0.5 & -0.4 & -0.5 & -0.5 & -1.8 & -1.4 & -3.2 \\
\hline$d_{5}$ & 0.8 & 0.6 & 0.5 & -0.5 & -0.8 & 0.1 & 1.9 & 1.2 & 3.1 \\
\hline$d_{6}$ & 0.3 & -0.3 & -0.5 & -0.5 & -0.4 & -0.5 & -0.5 & -1.4 & -1.9 \\
\hline
\end{tabular}

$$
\begin{aligned}
& { }^{T_{1}} \underline{\lambda_{1}+\lambda_{2} p}=\left({ }^{\mu_{T_{1}}} \underline{\lambda_{1}+\lambda_{2}} p^{\prime}{ }^{\gamma_{T_{1}}} \underline{\lambda_{1}+\lambda_{2}} p\right) \text { (given in Table 13) } \\
& T_{1}{\overline{\lambda_{1}+\lambda_{2}}}^{p}=\left(\mu_{T_{1}}{\overline{\lambda_{1}+\lambda_{2}}}^{p}, \gamma_{T_{1}}{\overline{\lambda_{1}+\lambda_{2}}}^{p}\right. \text { ) (given in Table 14), where }
\end{aligned}
$$

Table 13. Pessimistic multigranulation lower approximations of $T_{1}$.

\begin{tabular}{lllll}
\hline${ }^{\mu_{T_{1}}} \underline{\lambda_{\mathbf{1}}+\lambda_{2}} p^{\prime}{ }^{\gamma_{T_{1}}} \underline{\lambda_{\mathbf{1}}+\lambda_{\mathbf{2}}} p$ & $f_{\mathbf{1}}$ & $f_{2}$ & $f_{3}$ \\
\hline$\mu_{T_{1}} \underline{\lambda_{1}+\lambda_{2}} p\left(e_{1}\right),{ }^{\gamma T_{1}} \underline{\lambda_{1}+\lambda_{2}} p\left(e_{1}\right)$ & $(0.9,0.0)$ & $(0.9,0.0)$ & $(0.9,0.3)$ \\
\hline$\mu_{T_{1}} \underline{\lambda_{1}+\lambda_{2}} p\left(e_{2}\right),{ }^{\gamma T_{1}} \underline{\lambda_{1}+\lambda_{2}} p\left(e_{2}\right)$ & $(0.9,0.0)$ & $(0.8,0.2)$ & $(0.7,1)$ \\
\hline$\mu_{T_{1}} \underline{\lambda_{1}+\lambda_{2}} p\left(e_{3}\right),{ }^{\gamma T_{1}} \underline{\lambda_{1}+\lambda_{2}} p\left(e_{3}\right)$ & $(0.9,0.0)$ & $(0.6,1)$ & $(0.9,0.1)$ \\
\hline
\end{tabular}

Table 13 shows the exact degree of the competency of the applicant $f_{i}$ to $T_{1}$ in their batting experience, bowling experience and managerial expertise.

Table 14. Pessimistic multigranulation upper approximations of $T_{1}$.

\begin{tabular}{clll}
\hline$\mu_{T_{1}}{\overline{\lambda_{\mathbf{1}}+\lambda_{\mathbf{2}}}}^{p}, \gamma_{T_{1}}{\overline{\lambda_{\mathbf{1}}+\lambda_{\mathbf{2}}}}^{p}$ & $f_{\mathbf{1}}$ & $f_{2}$ & $f_{3}$ \\
\hline$\mu_{T_{1}}{\overline{\lambda_{1}+\lambda_{2}}}^{p}\left(e_{1}\right),{ }^{\gamma_{T_{1}}}{\overline{\lambda_{1}+\lambda_{2}}}^{p}\left(e_{1}\right)$ & $(0.9,0.2)$ & $(0.3,0.6)$ & $(0.6,0.6)$ \\
\hline$\mu_{T_{1}}{\overline{\lambda_{1}+\lambda_{2}}}^{p}\left(e_{2}\right),{ }^{\gamma_{T_{1}}}{\overline{\lambda_{1}+\lambda_{2}}}^{p}\left(e_{2}\right)$ & $(0.3,0.6)$ & $(0.7,0.2)$ & $(0.0,0.3)$ \\
\hline$\mu_{T_{1}}{\overline{\lambda_{1}+\lambda_{2}}}^{p}\left(e_{3}\right),{ }^{\gamma_{T_{1}}}{\overline{\lambda_{1}+\lambda_{2}}}^{p}\left(e_{3}\right)$ & $(0.3,0.6)$ & $(0.0,0.3)$ & $(0.6,0.3)$ \\
\hline
\end{tabular}

Table 14 shows the possible degree of competency of applicant $f_{i}$ to $T_{1}$ in their batting experience, bowling experience and managerial expertise.

It is shown in Table 15 that $S\left(f_{1}\right)=2.8$ is the maximum, so PCB will select applicant $f_{1}$.

Table 15. Values of score function of applicants.

\begin{tabular}{llllllllll}
\hline & $\underline{S}_{i j}\left(e_{1}\right)$ & $\underline{S}_{i j}\left(e_{2}\right)$ & $\underline{S}_{i j}\left(e_{3}\right)$ & $\bar{S}_{i j}\left(e_{1}\right)$ & $\bar{S}_{i j}\left(e_{2}\right)$ & $\bar{S}_{i j}\left(e_{3}\right)$ & $\underline{S}\left(x_{i}\right)$ & $\bar{S}\left(x_{i}\right)$ & $S\left(x_{i}\right)$ \\
\hline$f_{1}$ & 0.9 & 0.9 & 0.9 & 0.7 & -0.3 & -0.3 & 2.7 & 0.1 & 2.8 \\
\hline$f_{2}$ & 0.9 & 0.9 & -0.4 & -0.3 & 0.5 & -0.3 & 1.4 & -0.1 & 1.3 \\
\hline$f_{3}$ & 0.6 & -0.3 & 0.8 & 0.0 & -0.3 & 0.3 & 1.1 & 0.0 & 1.4 \\
\hline
\end{tabular}

\section{Comparison}

The MGRS of IFS based on multi soft relations is needed in many real world problems because it is an admired reality that a collective decision based on multiple experts' opinions is wiser than a single expert's opinion. If we discuss the probability RS model [51], it is clear that there are three problems needs to be managed. Firstly, the interpretations and calculations of the thresholds are very poor. Secondly, there is a lack of an estimation of the conditional probability. Thirdly, there is no valid interpretation of the positive, negative and boundary region with probability thresholds. To solve these problems, a decision theoretic 
RS model [52] has been proposed. Then, a MGRS model in terms of equivalence relations has been proposed, which is a better model to deal with incompleteness and vagueness. However, we still had problems due to the equivalence relation. It was necessary to relax the conditions of the equivalence relation. Shabir et al. [40] introduced a MGRS model with crisp sets in terms of soft relations. After that, they proposed the OMGFRS using a FS instead of a crisp set [50]. Recently, Shabir et al. [41] gave the extension of the OMGFRS model of an IFS and presented the OMGIFRS model in terms of soft relations to prepare a wiser decision in decision-making problems. An IFS is better than a crisp set or a FS to discuss the uncertainty. In this paper, we investigate the pessimistic MGRS of the IFS in terms of soft binary relations. Our proposed model, the PMGIFRS, is more capable in dealing with uncertainty because of the presence of the non-membership degree in the IFS. Furthermore, soft relations have more applications than crisp relations in managing uncertainty due to its parameterization of binary relations. In our proposed PMGIFRS model, we used an IFS instead of a crisp set. A crisp set does not discuss the uncertainty and incompleteness in our daily life problems. However, the IFS discusses the uncertainty, vagueness and incompleteness with the concept of the degree of membership, degree of non-membership and degree of hesitance. In comparison with IF formalism and nonIF formalism, we can see in our proposed model that IF formalism is better than non IF formalism.

\section{Conclusions}

This article presents the PMGRS model of an IFS in terms of a soft relation over dual universes. Firstly, we described the MGRS in terms of two soft relations using IFSs regarding the aftersets and foresets, and the resulting sets are two IFSSs regarding the aftersets and foresets. After that, we studied some algebraic properties fo the proposed PMGRS model. Then, we generalized this MGRS model to the granulation roughness of an IFS in terms of multi soft relations and discussed some of its properties. At the end, an example is presented that illustrates the importance and significance of the proposed decision algorithm. In future, the OMGRIFS and PMGRIFS will be discussed in terms of extended forms of a FS and IFS. Our proposed model, the PMGIFRS, has an advantage over other existing models, which is that we can make an approximation of an IFS of a universe in some other universe and can make decisions based on each parameter. These models may be studied in terms of pythagorean FSs, basic uncertain information SSs and liguistic Z-number FSSs.

Author Contributions: Conceptualization: S.B. and M.S.; methodology: M.Z.A.; software: S.B.; validation: A.N.A.-K.; formal analysis: M.Z.A.; investigation: M.Z.A.; resources: A.N.A.-K.; data curation: M.S.; writing-original draft preparation: M.Z.A.; writing—review and editing: S.B.; visualization: M.Z.A.; supervision: S.B.; project administration: M.Z.A.; funding acquisition: A.N.A.-K.; All authors have read and agreed to the published version of the manuscript.

Funding: This research received no external funding.

Institutional Review Board Statement: Not applicable.

Informed Consent Statement: Not applicable.

Data Availability Statement: We did not use any data for this research work.

Conflicts of Interest: The authors declare that they have no conflict of interest. 


$\begin{array}{ll}\text { Abbreviations } \\ \text { Acronyms } & \text { Representations } \\ \text { FSs } & \text { Fuzzy sets } \\ \text { IFSs } & \text { Intuitionistic fuzzy sets } \\ \text { RSs } & \text { Rough sets } \\ \text { SSs } & \text { Soft sets } \\ \text { FSSs } & \text { Fuzzy soft sets } \\ \text { IFSSs } & \text { Intuitionistic fuzzy soft sets } \\ \text { SRSs } & \text { Soft rough sets } \\ \text { IFRSs } & \text { Intuitionistic fuzzy rough sets } \\ \text { FRSs } & \text { Fuzzy rough sets } \\ \text { RFSs } & \text { Rough fuzzy sets } \\ \text { MGRS } & \text { Multigranulation rough set } \\ \text { OMGRS } & \text { Optimistic multigranulation rough set } \\ \text { PMGRS } & \text { Pessimistic multigranulation rough set } \\ \text { IFMGRS } & \text { Intuitionistic fuzzy multigranulation rough set } \\ \text { MCGDM } & \text { Multi-criteria group decision making } \\ \text { OMGFRS } & \text { Optimistic multigranulation fuzzy rough set } \\ \text { OMGIFRS } & \text { Optimistic multigranulation intuitionistic fuzzy rough set } \\ \text { IFN } & \text { Intuitionistic fuzzy number } \\ \text { IFV } & \text { Intuitionistic fuzzy value }\end{array}$

\section{References}

1. Zadeh, L.A. Fuzzy sets. Inform. Control. 1965, 8, 338-353. [CrossRef]

2. Atanassov, K.T. Intuitionistic fuzzy sets. Fuzzy Sets Syst. 1986, 20, 87-96. [CrossRef]

3. Ejegwa, P.A.; Onoja, A.M.; Emmanuel, I.T. A note on some models of intuitionistic fuzzy sets in real life situations. J. Glob. Res. Math. Arch. 2014, 2, 42-50.

4. Molodtsov, D. Soft set theory-First results. Comput. Math. Appl. 1999, 37, 19-31. [CrossRef]

5. Xu, W.; Ma, J.; Wang, S.; Hao, G. Vague soft sets and their properties. Comput. Math. Appl. 2010, 59, 787-794. [CrossRef]

6. Maji, P.K.; Biswas, R.; Roy, A.R. Fuzzy soft sets. J. Fuzzy Math. 2001, 9, 589-602.

7. Maji, P.K.; Biswas, R.; Roy, A.R. Intuitionistic fuzzy soft sets. J. Fuzzy Math. 2001, 9, 677-692.

8. Ali, M.I. A note on soft sets, rough soft sets and fuzzy soft sets. Appl. Soft Comput. 2011, 11, 3329-3332.

9. Xiao, Z.; Xia, S.; Gong, K.; Li, D. The trapezoidal fuzzy soft set and its application in MCDM. Appl. Math. Model. 2012, 36, 5844-5855. [CrossRef]

10. Sang, M.Y.; Seok, J.L. New approach to intuitionistic fuzzy rough sets. Int. J. Fuzzy Log. Intell. Syst. 2020, 20, 129-137.

11. Pawlak, Z. Rough sets. Int. J. Comput. Inform. Sci. 1982, 11, 341-356. [CrossRef]

12. Zhang, Q.; Xie, Q.; Wang, G. A survey on rough set theory and its applications. CAAI Trans. Intell. Technol. 2016, 1, 323-333. [CrossRef]

13. Yao, Y.Y.; Greco, S.; Słowiński, R. Probabilistic rough sets. In Springer Handbook of Computational Intelligence; Springer: Berlin/Heidelberg, Germany, 2015; pp. 387-411.

14. Ziarko, W. Variable precision rough set model. J. Comput. Syst. Sci. 1993, 46, 39-59. [CrossRef]

15. Herbert, J.P.; Yao, J.T. Game-theoretic rough sets. Fundam. Inf. 2011, 108, 267-286. [CrossRef]

16. Lashin, E.F.; Kozae, A.M.; Abo Khadra, A.A. Rough set theory for topological spaces. Int. J. Approx. Reason. 2005, 40, 35-43. [CrossRef]

17. Chen, Y.M.; Miao, D.Q.; Wang, R.Z. A rough set approach to feature selection based on ant colony optimization. Pattern Recognit. Lett. 2010, 31, 226-233. [CrossRef]

18. Qin, Z.G.; Mao, Z.Y.; Deng, Z.Z. Review of genre-based teaching approaches. J. South China Univ. Technol. 2000, $28,30-34$.

19. Slezak, D. Rough Sets and Few-Objects-Many-Attributes Problem: The Case Study of Analysis of Gene Expression Data Sets; IEEE: Piscataway, NJ, USA, 2007; pp. 497-500.

20. Zhou, Z.Y.; Shen, G.Z. Application of rough set theory in determining index weight of intelligence analysis. Inf. Stud. Theory Appl. 2012, 35, 61-65.

21. Pérez-Díaz, N.; Ruano-Ordás, D.; Méndez, J.R. Rough sets for spam filtering: Selecting appropriate decision rules for boundary e-mail classification. Appl. Soft Comput. 2012, 12, 3671-3682. [CrossRef]

22. Qian, Y.H.; Liang, J.H.; Yao, Y.Y. MGRS: A multi-granulation rough set. Inf. Sci. 2010, 180, 949-970. [CrossRef]

23. Qian, Y.H.; Liang, J.Y.; Yao, Y.Y.; Dang, C.Y. Incomplete mutigranulation rough set. IEEE Trans. Syst. Man. Cybern. Part A 2010, 20, 420-430. [CrossRef]

24. Lin, G.P.; Liang, J.Y.; Qian, Y.H. Multigranulation rough sets: From partition to covering. Inf. Sci. 2013, 241, 101-118. [CrossRef] 
25. Xu, W.H.; Zhang, W.X. Measuring roughness of generalized rough sets induced by a covering. Fuzzy Sets Syst. 2007, 158, $2443-2455$. [CrossRef]

26. Xu, W.; Sun, W.; Zhang, X.; Zhang, W. Multiple granulation rough set approach to ordered information systems. Int. J. Gen. Syst. 2012, 41, 475-501. [CrossRef]

27. Xu, W.H.; Wang, Q.R.; Zhang, X.T. Multi-granulation rough sets based on tolerance relations. Soft Comput. 2013, 17, 1241-1252. [CrossRef]

28. Huang, B.; Wu, W.Z.; Yan, J.; Li, H.; Zhou, X. Inclusion measure-based multi-granulation decision-Theoretic rough sets in multi-scale intuitionistic fuzzy information tables. Inf. Sci. 2020, 507, 421-448. [CrossRef]

29. Liang, M.; Mi, J.; Feng, T.; Xie, B. A dynamic approach for updating the lower approximation in adjustable multi-granulation rough sets. Soft Comput. 2020, 24, 15951-15966. [CrossRef]

30. Shao, Y.; Qi, X.; Gong, Z. A general framework for multi-granulation rough decision-making method under q-rung dual hesitant fuzzy environment. Artif. Intell. Rev. 2020, 53, 4903-4933. [CrossRef]

31. Kong, Q.; Zhang, X.; Xu, W.; Xie, S. Attribute reducts of multi-granulation information system. Artif. Intell. Rev. 2019, 53, 1353-1371. [CrossRef]

32. Sun, L.; Wang, L.; Ding, W.; Qian, Y.; Xu, J. Neighborhood multi-granulation rough sets-based attribute reduction using lebesgue and entropy measures in incomplete neighborhood decision systems. Knowl.-Based Syst. 2020, 192, 105373. [CrossRef]

33. Wong, S.K.M.; Wang, L.S.; Yao, Y.Y. On modeling uncertainty with interval structures. Comput. Intell. 1995, 11, 406-426. [CrossRef]

34. Liu, G.L. Rough set theory based on two universal sets and its applications. Knowl.-Based Syst. 2010, 23, 110-115. [CrossRef]

35. Huang, B.; Guo, C.; Zhuang, Y.1.; Li, H.; Zhou, X. Intuitionistic fuzzy multigranulation rough sets. Inf. Sci. 2014, 277, 299-320. [CrossRef]

36. Pang, J.; Guan, X.; Liang, J.; Wang, B.; Song, P. Multi-attribute group decision-making method based on multi-granulation weights and three-way decisions. Int. J. Approx. Reason. 2020, 117, 122-147. [CrossRef]

37. Sun, B.Z.; Ma, W.M. Multigranulation rough set theory over two universes. J. Intell. Fuzzy Syst. 2015, 28, 1251-1269. [CrossRef]

38. Tan, A.; Wu, W.Z.; Shi, S.; Zhao, S. Granulation selection and decision making with multigranulation rough set over two universes. Int. J. Mach. Learn. Cybern. 2018, 10, 2501-2513. [CrossRef]

39. Xu, Z. Intuitionistic preference relations and their application in group decision making. Inf. Sci. 2007, 177, 2363-2379. [CrossRef]

40. Shabir, M.; Din, J.; Ganie, I.A. Multigranulation roughness based on soft relations. J. Intell. Fuzzy Syst. 2021, 40, 10893-10908. [CrossRef]

41. Anwar, M.Z.; Bashir, S.; Shabir, M.; Alharbi, M.G. Multigranulation roughness of intuitionistic fuzzy sets by soft relations and their applications in decision making. Mathematics 2021, 9, 2587. [CrossRef]

42. Shabir, M.; Ayub, S.; Bashir, S. Prime and Semiprime L-Fuzzy Soft Bi-Hyperideals. J. Hyperstruct. 2017, 6, 109-119.

43. Shabir, M.; Ayub, S.; Bashir, S.Applications of L-Fuzzy soft sets in semihypergroups. J. Adv. Math. Stud. 2017, 10, 367-385.

44. Chen, S.M.; Tan J.M. Handling multicriteria fuzzy decision-making problems based on vague set theory. Fuzzy Sets Syst. 1994, 67, 163-172. [CrossRef]

45. Hong, D.H.; Choi, C.H. Multi-criteria fuzzy decision-making problems based on vague set theory. Fuzzy Sets Syst. 2000, 114, 103-113. [CrossRef]

46. Xu, Y.; Sun, Y.; Li, D. Intuitionistic fuzzy soft set. In Proceedings of the 2nd International Workshop on Intelligent Systems and Applications, Wuhan, China, 22-23 May 2010; IEEE: Piscataway, NJ, USA, 2010; pp. 1-4.

47. Feng, F.; Ali, M.I.; Shabir, M. Soft relations applied to semigroups. Filomat 2013, 27, 1183-1196. [CrossRef]

48. Li, Z.; Xie, N.; Gao, N. Rough approximations based on soft binary relations and knowledge bases. Soft Comput. 2017, 21, 839-852. [CrossRef]

49. Anwar, M.Z.; Bashir, S.; Shabir, M. An efficient model for the approximation of intuitionistic fuzzy sets in terms of soft relations with applications in decision making. Math. Probl. Eng. 2021, 2021, 6238481. [CrossRef]

50. Jamal, D.; Shabir, M. Multigranulation roughness of a fuzzy set based on soft relations over dual universes and its application. Mathematics 2021, submitted.

51. Li, H.X.; Zhou, X.Z.; Li, T.R. Decision-Theoretic Rough Sets Theory Sets Theory and Its Application; Science Press: Beijing, China, 2011.

52. Yu, H.; Wang, G.Y.; Yao, Y.Y. Current research and future perspectives on decision-theoretic rough sets. Chin. J. Comput., 2015, 8, 1628-1639. 